\title{
A Study of Lubricant Film Thickness in Compliant Contacts of Elastomeric Seal Materials Using a Laser Induced Fluorescence Technique
}

\author{
M.T. Fowell ${ }^{1}$, C. Myant ${ }^{1}$, H.A. Spikes ${ }^{1}$, A. Kadiric $^{1 *}$ \\ ${ }^{1}$ Tribology Group, Imperial College London, London, SW7 2AZ, UK \\ *Corresponding Author: a.kadiric@imperial.ac.uk
}

\begin{abstract}
A laser induced fluorescence technique was used to investigate the build-up of lubricant films in compliant contacts operating in the isoviscous elasto-hydrodynamic regime (I-EHL). The described technique utilises an optimised optical set-up with a relatively high signal-to-noise ratio and was shown to be able to produce film thickness maps of the complete contact area and measure a very wide span of thicknesses, from $50 \mathrm{~nm}$ to $100 \mu \mathrm{m}$. Maps of film thickness were obtained over a range of entrainment speeds and loads for three different contact configurations and two elastomer materials, polydimethylsiloxane (PDMS) and a fluorocarbon rubber (FKM) which is typically used in rotary seal applications. In a model contact of a nominally smooth PDMS ball sliding on a glass flat, a crescent shaped area of reduced film thickness was observed towards the contact exit. In contrast to typical elasto-hydrodynamic contacts, no side-lobes of reduced film thickness were recorded, while the central film region exhibited a converging wedge shape. The elliptical contact of an FKM O-ring rolling on a glass flat showed a central region of flat film while areas of minimum film thickness were located near the contact edges either side of the centre. The highly conformal contact of relatively rough FKM O-ring sliding against a concave glass lens, a geometry more representative of that found in elastomeric seals, showed discrete regions of reduced film, corresponding to surface roughness asperities. With rising entrainment speed, some lift-off was observed, with surface roughness asperities appearing to be increasingly compressed. Measured films thicknesses were compared to existing theoretical predictions for I-EHL contacts and the level of agreement was found to be highly dependent on contact geometry and applied conditions.
\end{abstract}

Keywords: Seals, Elastomers, Compliant Contacts, Film thickness, Rolling Bearings, Isoviscous, EHL, LIF, Fluorescence

\section{Nomenclature}

$\begin{array}{llll}a & \text { Contact semi width in x plane }(\mathrm{m}) & h_{o} & \text { Outlet film thickness }(\mathrm{m}) \\ b & \text { Contact semi width in y plane }(\mathrm{m}) & k & \text { Ellipticity parameter }(\mathrm{a} / \mathrm{b}) \\ h_{c} & \text { Central Film Thickness }(\mathrm{m}) & r_{x 1} & \text { Radius of body } 1 \text { in x plane }(\mathrm{m}) \\ h_{i} & \text { Inlet film thickness }(\mathrm{m}) & r_{x 2} & \text { Radius of body } 2 \text { in x plane }(\mathrm{m}) \\ h_{m} & \text { Minimum film thickness }(\mathrm{m}) & r_{y 1} & \text { Radius of body } 1 \text { in y plane }(\mathrm{m})\end{array}$




\begin{tabular}{|c|c|c|c|}
\hline$r_{y 2}$ & Radius of body 2 in y plane $(\mathrm{m})$ & $M$ & Dimensionless load parameter (Mo \\
\hline$u$ & Entrainment speed $(\mathrm{m} / \mathrm{s})$ & $R_{x}^{\prime}$ & Reduced radius in $\mathrm{x}$ plane $(\mathrm{m})$ \\
\hline$w$ & Load $(N)$ & $R_{y}^{\prime}$ & Reduced radius in y plane $(\mathrm{m})$ \\
\hline$x$ & $x$ co-ordinate $(m)$ & $\bar{U}$ & Dimensionless Speed Parameter \\
\hline$y$ & y co-ordinate (m) & $\bar{W}$ & Dimensionless Speed Parameter \\
\hline C & Geometric constant (Moes) & $\varepsilon$ & Molar absorption coefficient $\left(\mathrm{M}^{-1}\right)$ \\
\hline$C_{d y e}$ & Concentration of dye (M) & $\eta$ & Viscosity (Pa s) \\
\hline$D$ & Reduced radius of curvature (Moes) & $\lambda_{e x}$ & Excitation wavelength $(\mathrm{nm})$ \\
\hline$E_{1}$ & Elastic modulus of body $1(\mathrm{~Pa})$ & $\lambda_{\text {em }}$ & Emission wavelength $(\mathrm{nm})$ \\
\hline$E_{2}$ & Elastic modulus of body $2(\mathrm{~Pa})$ & $v_{1}$ & Poisson ratio of body 1 \\
\hline$E^{\prime}$ & Reduced elastic modulus ( $\mathrm{Pa}$ ) & $v_{2}$ & Poisson ratio of body 2 \\
\hline$I_{e x}$ & Light intensity at exciting wavelength & $\Phi$ & Quantum efficiency \\
\hline$I_{f}$ & Light intensity of emitted wavelength & & \\
\hline$K_{h y}$ & Hydrodynamic convergence ratio & & \\
\hline
\end{tabular}

\section{Introduction}

Rolling element bearings often contain elastomer rotary lip seals to prevent contamination of the lubricant, usually grease, with water and debris in order to increase product life and re-lubrication interval. With the addition of seals many bearings have been shown to have virtually infinite life, however the presence of a seal increases frictional torque and therefore reduces overall machine efficiency. To improve machine efficiency and reliability it is therefore important to understand the operation of lubricated sliding contacts between elastomers and rigid counterfaces. However, the nature of lubricating film build up in compliant elastomer contacts under sliding conditions is not well understood. Furthermore, the contact geometry between a bearing lip seal and a raceway is nominally conformal and parallel in nature. It, therefore, lacks the usual converging nature at the inlet associated with hydrodynamic contacts meaning that no obvious lubricant entrainment mechanism exists. Despite this, it has been understood for a long time that lubricant is present between the surfaces. Some of the first direct experimental evidence for this was provided by Ogata et al. [1], although it had been "common experience" for many years [2]. For the seals in question Ogata et al. [1] note that the full range of lubrication regimes from boundary, through mixed and $\mathrm{EHL}$, to full film hydrodynamic lubrication could be observed over a wide range of speeds (1-7000 rpm). The presence of a thin lubricant film in such contacts plays a key role in influencing contact friction, particularly under mixed or partial lubrication conditions, and in controlling wear of the contacting bodies and therefore the life of the components. The current study is an attempt to 
provide better understanding of the build-up of fluid films in lubricated contacts between a compliant elastomer body and a rigid counter face, with emphasis on materials and sliding conditions representative of those found in bearing seals.

\section{Background}

Elastomer contacts exist in many engineering and biological applications. In addition to bearing seals [3], typical examples include windscreen wipers [4], tyres [5, 6], artificial joints [7] and foodstuffs [8]. In many of these applications a lubricating fluid is present in the contact. In such cases the problem is characterised by pressures which are of a magnitude that produces significant elastic deformation of the compliant bodies but is not sufficient to cause a significant increase in the lubricant viscosity and bulk modulus. Consequently, this lubrication regime is termed isoviscous elasto-hydrodynamic lubrication (I-EHL) or "soft elasto-hydrodynamic" lubrication. Research into the behaviour of frictional conjunctions formed by bodies with low elastic modulus (<1 GPa) such as elastomers first began in earnest in the late 1950s [1]. The following 25 years saw a number of significant studies on elastomer contacts, both lubricated and dry, including the effects of material properties such as substrate viscoelasticity and surface adhesion [9-21].

In case of hard contacts, both experiments and models based on the Reynolds equation have been used to establish the well-known empirical relationships between the central and minimum film thicknesses, $h_{c}$ and $h_{m}$ respectively, and the key operating parameters (speed, applied load, viscosity and material properties) based on regression analysis [22]. For materials of low elastic modulus, the relevant experimental data is scarce and such relationships are much less common. The most widely used of these are the equations produced by Hamrock and Dowson [23] based on numerical EHL solutions of compliant contacts:

$h_{c}=7.32\left(1-0.72 e^{-0.28 k}\right) \bar{U}^{0.64} \bar{W}^{-0.22} R_{x}{ }^{\prime}$

$h_{m}=7.43\left(1-0.85 e^{-0.31 k}\right) \bar{U}^{0.66} \bar{W}^{-0.21} R_{x}{ }^{\prime}$

where $k$ is the ellipticity parameter which reduces to 1 for point or circular contacts and $\bar{U}, \bar{W}$ are the dimensionless speed and load parameter respectively, and are defined as:

$\bar{U}=\frac{u \eta}{E \prime R_{x^{\prime}}}$

$\bar{W}=\frac{w}{E \prime R_{x^{\prime}}}$

where $u$ is the entrainment speed, $w$ the applied load, $\eta$ the lubricant dynamic viscosity, $R_{x}{ }^{\prime}$ the reduced radius of curvature in the entrainment direction, and $E^{\prime}$ the reduced elastic modulus. The latter two terms are defined by $1 / R_{x}^{\prime}=1 / r_{x 1}+1 / r_{x 2}$ and $2 / E^{\prime}=\left(1-v_{1}^{2}\right) / E_{1}+\left(1-v_{2}^{2}\right) / E_{2}$, respectively, where $r_{x 1}, r_{x 2}, E_{1}, E_{2}, v_{1}$, and $v_{2}$ denote the radii in the entrainment direction, the Young's moduli, and the Poisson's ratios of the two contacting bodies respectively. A limited number of other authors have derived similar equations [24-26]. In addition to the Hamrock and Dowson's model, the present paper will also utilise the regression fit produced by Nijenbanning et al. [25] for the central film thickness, $h_{c}$, in an isoviscous elastic contact.. This model is based on Moes parameters $(M, L)$ for non-dimensionalisation of the EHL lubrication problem [27]. The central film thickness equation, $h_{c}$, from this model is shown below in equations (5-6). 
$h_{c}=C_{c}^{E I}(D) M^{-2 / 15}$

In which,

$C_{c}^{E I}(D)=3.18\left(1+0.006 \ln D+0.63 D^{4 / 7}\right)^{-14 / 25} D^{-1 / 15}$

where $M$ is the dimensionless Moes load parameter, $L$ is the dimensionless Moes lubricant parameter, which is equal to zero for the case of an isoviscous contact. $D$ is a measure of the ratio of the reduced radii in the $x$ and $y$ planes and is related to the ellipticity parameter used by Hamrock and Dowson [23]. The parameters $M$ and $D$ are defined as follows:

$M=\bar{W} \bar{U}^{-3 / 4}$

$D=R^{\prime}{ }_{x} / R^{\prime}{ }_{y}$

While in hard contacts it has long been possible to experimentally validate these empirical fits, in soft or compliant contacts obtaining the measurements of film thickness required for such validation has proved challenging. Optical interferometry [13, 18, 3], magnetic resistance [28], laser induced fluorescence (LIF) [29-34], Raman spectroscopy [35] and ultrasonic reflection [36] techniques have all been applied to measurement of thin films present in compliant lubricated tribological contacts with varying degree of success. However, each of these techniques has limitations and difficulties due to characteristics of lubricated elastomer contacts including:

- Lubricant film thickness in I-EHL contacts covers a wide range of values, from fractions to the hundreds of microns and the required measuring range of the chosen technique needs to be appropriately large.

- Contact size is considerably larger than in non-compliant (hard) tribological contacts.

- Elastomer components have a high roughness compared to surfaces usually investigated with established techniques. They are also very difficult to polish.

- Elastomer materials are poor reflectors of light while coating soft surfaces with reflective layers is not trivial as the applied coatings are either prone to wear or modify the compliance and surface properties significantly, or both.

Two particular studies utilising laser induced fluorescence techniques to measure the film thickness in compliant contacts are worth noting further. Myant et al. [29] used the model system of a PDMS hemisphere loaded against a glass flat to study the build-up of a lubricant film formed by glycerolwater solutions under pure sliding conditions. They considered model contacts with relatively low speeds, up to $1.6 \mathrm{~mm} / \mathrm{s}$ and very smooth PDMS surfaces. The experimental setup used was limited by noise to measurements of film thicknesses above $300 \mathrm{~nm}$. At the highest glycerol concentration they noted a discrepancy between the measured film thickness and that predicted by Hamrock and Dowson [23], which they attributed to a drop in the viscosity of the glycerol caused by its hygroscopic nature. Poll et al. [32] employed a similar fluorescence method in a study of an actual rotary lip seal running against a glass cylinder at low speeds (up to $100 \mathrm{~mm} / \mathrm{s}$ ). They found that average film thickness remained constant with varying entrainment speed. While the authors stated that the theoretical resolution of the technique was of the order of $20 \mathrm{~nm}$, the real resolution of any 
LIF technique is ultimately limited by the signal-to-noise ratio achievable by the chosen experimental set-up. Thus the minimum film thickness measured in their study was around $350 \mathrm{~nm}$. The observation of constant film thickness with rising surface speed suggests the absence of a hydrodynamic lift-off; however, it could also be a result of entrapped lubricant within roughness features or experimental noise limiting the real resolution.

The presence of an adequate lubricant film in seals, and other elastomer contacts, is critical to the frictional and wear performance of the seal and the component in which it is installed. Therefore the development of a reliable film measuring method that is able to overcome some of the problems outlined above is of practical importance. As yet there is no established technique for routinely obtaining film thickness data from a soft contact and, in particular one which is able to cope with the conditions, materials, and surface roughnesses pertinent to rotary seals. In this study an improved Laser Induced Fluorescence (LIF) technique has been developed which has sufficient resolution and accuracy to become routine. The technique has been used to observe the film build-up and to measure the film thickness in conformal and non-conformal, soft elastomer contacts, including those where materials, and their associated surface roughness, are representative of bearing seal contacts. This has been made possible through the improved spatial resolution in the $x-y$ plane of the current technique, high signal-to-noise ratio and the ability to measure thin films so that film thicknesses in the vicinity of roughness features can be accurately measured. This in turn allows for better understanding of liquid film lubrication under realistic elastomeric seal conditions as well as general compliant contacts.

Tests were carried out over a wide range of entrainment speeds for a non-conformal polydimethylsiloxane (PDMS) hemisphere and FKM fluoro-elastomer on glass contacts with different levels of conformity. The observed results are discussed in terms of mechanisms of lubricant film build-up and observed film shape, and compared with theoretical predictions for film thickness in isoviscous elasto-hydrodynamic contacts.

\section{$4 \quad$ Experimental Setup for Film Thickness Mapping}

\subsection{Application of Laser Induced Fluorescence for Measuring Thin Lubricant Films}

Fluorescence is a special case of luminescence where a photon of light is emitted from an electronically-excited state of a molecule, the fluorescent dye. Laser Induced Fluorescence (LIF) uses a laser as a means of providing the energy, in the form of incident photons, required for this excitation process to occur. Energy from the exciting photon causes an electron within the dye molecule to transition from the ground state to the excited state. Subsequently, this excited electron decays back to the ground state by emitting a photon which is red-shifted (i.e. towards longer wavelength) with respect to the exciting wavelength. This phenomenon is known as the Stokes shift [37]. Therefore, for a given volume of dye containing fluid the magnitude of emitted photons is a function of the number of fluorescent species present (concentration), $C_{d y e}$, the number of incident photons (excitation intensity), $I_{e x}$, the probability of absorption of an incident photon by a dye molecule (molar absorption coefficient), $\varepsilon\left(\lambda_{e x}\right)$, the number of absorbed molecules which result in emitted photons (quantum yield), $\Phi\left(\lambda_{e m}\right)$, and the path length of the incident light beam through the dye containing solution, $k$. Using these values a formal expression for the emitted fluorescent intensity at low concentrations can be derived from the Beer-Lambert Law, equation (9). 


$$
I_{f}\left(\lambda_{e x}, \lambda_{e m}\right)=2.303 k I_{e x} \varepsilon\left(\lambda_{e x}\right) \Phi\left(\lambda_{e m}\right) C_{d y e}
$$

From equation (9) it is clear that if the concentration of dye is kept constant then the ratio of the incident excitation intensity and the emitted intensity is proportional to the optical path length provided that no fluorescent concentration quenching or photo-bleaching takes place. Therefore, when these conditions are met, it is possible to measure the thickness of a lubricant film containing fluorescent species by measurement of this ratio and the knowledge of incident excitation intensity.

In the current study, the test lubricant (75\% wt. glycerol, $25 \%$ wt. water) was doped with $0.01 \%$ wt. of Rhodamine $6 \mathrm{G}$ fluorescent dye which is equivalent to a molar concentration of dye of $250 \mu \mathrm{M}$.

\subsubsection{Influence of Fluorescent Quenching}

No fluorescent concentration quenching occurs with the chosen set-up because the selected concentration of dye is well below the value of $9 \mathrm{mM}$ at which it has been shown that concentration quenching effects start to take place in methanol through the formation of aggregates and excimers [38].

\subsubsection{Influence of Temperature on Fluorescence}

Temperature is known to affect the fluorescent intensity of Rhodamine $6 \mathrm{G}$ in a similar way to other Xanthene dyes [39]. In the case of Rhodamine $6 \mathrm{G}$ a reduction in emitted fluorescent intensity of $\sim 20 \%$ is observed in glycerol for a rise of $50^{\circ} \mathrm{C}$ in bulk solution temperature. In the current study calibration is performed at each test temperature and the solution supplied to the radiated area in a large enough quantity to assume that no appreciable rise in temperature occurs.

\subsubsection{Influence of Viscosity on Fluorescence}

Viscosity dependence of the fluorescence intensity was checked by measurement of spectra for 6 test fluids ranging from $50 \%$ water , $50 \%$ glycerol solution to $15 \%$ water , $85 \%$ glycerol solution each with a dye concentration of $2.5 \mu \mathrm{M}$. This selection of test fluids spanned a viscosity range of $6 \mathrm{mPa} \mathrm{s}$ to $109 \mathrm{mPa}$ s. Intensity varied by $0.7 \%$ across this range in line with the dye concentration error.

\subsection{Laser Induced Fluorescence Optical Set-up}

A schematic of the present optical setup is shown in Figure 1. The fluorescence intensity images were observed with a reflected light microscope containing a filter cube with emission and excitation filters and a dichroic mirror. A $5 x$ objective was used and images were captured using a Electron Multiplying Gain CCD camera. Excitation was provided using a green $(532 \mathrm{~nm}) 400 \mathrm{~mW}$ solid-state, diode-pumped laser which was expanded (Fig. 1, label (2)) and refocused (Fig. 1, label (4)) at the back focal plane the objective to give epi-illumination.

\subsection{Contact Arrangements for Film Thickness Mapping}

Film thickness was measured in three different contact configurations:

1. The first set-up is a point contact formed by a hemispherical elastomer (PDMS) specimen loaded against a flat glass disc, as shown in Figure 2(a). The hemispherical specimen has radii of $12.7 \mathrm{~mm}$ in both the $x$ and $y$ planes. The bottom surface of the disc is immersed in lubricant so that the lubricant is entrained into the contact by rotation of the glass disc. Tests are conducted in pure sliding conditions. The glass disc has a surface roughness, $R_{a}<10 \mathrm{~nm}$, a Young's modulus of $65 \mathrm{GPa}$, Poisson's ratio of 0.24 and a refractive index of 1.517 . 
2. In the second configuration, an elliptical contact is formed between an elastomer O-ring (FKM) and a glass disc as shown in Figure 2(b). The O-ring is mounted on a barrelled roller with sufficient interference so as to prevent circumferential creep of the O-ring. The mounted O-Ring has radii of $10.4 \mathrm{~mm}$ in the $x$ plane and $1.31 \mathrm{~mm}$ in the $y$ plane respectively. The resulting contact has an ellipticity ratio of $a / b=3.9$, see Table 1 . In this scenario both the O-ring and the disc are rotated so that the contact is in pure rolling. The condition of pure rolling is of course not representative of a rotary lip seal but this configuration serves as a reference and provides additional understanding of lubrication mechanisms of soft EHL contacts.

3. In the third configuration, an elastomer O-ring (FKM), again mounted on a barrel roller, is loaded against a concave cylindrical BK7 glass lens as shown in Figure 2(c). The O-ring and the glass lens have similar radii in the entrainment direction, $-12.96 \mathrm{~mm}$ and 10.4 $\mathrm{mm}$ respectively, which results in a contact with a high level of conformity. The contact ellipse is highly extended in the $x$ direction, with ellipticity ratio $a / b=10.6$, see Table 1 . The high level of conformity is intended to more closely approximate the conformal contact of a rotary seal and a shaft. Although the seal-shaft contact is nominally fully conformal, when bearing is loaded or undergoes some vibration, the seal is located slightly off centre so that a small amount of non-conformity is introduced. However, even taking this into account the level of conformity in the current experimental set-up is still likely to be less than that in an operating seal, although it is more representative of the seal-shaft contact than most of the existing studies of elastomer lubrication and certainly more than the configurations 1 and 2 above. It should also be noted that the O-ring does not form a complete contact with the glass lens and the lower half of the seal is exposed and dip lubricated, which may allow some entrainment of lubricant within roughness features of the O-ring surface. Unfortunately the current setup does not allow for complete contact but this may be addressed in future work. Incomplete contact and the inherent slight non-conformity described above, may both contribute to lubricant entrainment in the present set-up in a manner which may not be present in an operating rotary lip seal. The reported results are therefore intended to provide further understanding of mechanisms at play and reveal trends in film build up, while the reported absolute values of the film thicknesses are only indicative of those that may exist between the elastomer seal and a shaft at given conditions. It should be noted that the glass discs and lenses used in the study have no reflective coatings.

Table 1 lists the applied contact conditions, specimen material properties and geometries of the three configurations studied. It should also be noted that the elliptical contacts studied in configurations 2 and 3 extend beyond the field of view of the optical set up due to the large ellipticity ratios. In these cases the field of view is centred towards the inlet of the conjunction so that the maximum proportion of the nominal static contact area is captured and shown in film thickness maps presented later.

\subsection{Elastomer Specimens}

The hemispherical elastomer samples were made from PDMS and were moulded in a plano-concave lens radius of $12.7 \mathrm{~mm}$. Carbon black (CB) was added to the PDMS at $0.5 \%$ wt. as filler to absorb 
transmitted light. Average surface roughness of the manufactured PDMS samples was measured using a white light interferometric optical profilometer and was found to be $R_{a}=10.3 \mathrm{~nm}$. The elastic modulus of the resulting elastomer was measured as $E=3.8 \mathrm{MPa}$ using dynamic mechanical analysis. It should be noted that the addition of carbon black to PDMS had negligible effect on the refractive index and therefore reflectivity of the material.

For contact configurations 2 and 3, custom O-ring samples were made from FKM rubber and constructed using extruded elastomer splicing cord. Constructing O-rings in this manner eliminates the pronounced mould lines present on ready-made O-rings, which might interfere with film formation. The FKM elastomer used had a Shore A hardness of 70. Average surface roughness of the tested samples was measured to be $R_{a}=800 \pm 50 \mathrm{~nm}$ and elastic modulus, $E=7.3 \mathrm{MPa}$.

\subsection{Experimental Procedure}

A new elastomer sample (hemisphere or O-Ring) was used for each test. The following cleaning procedure was used for both, the elastomer sample and the counter-face (glass disc or cylindrical lens):

1. Washing in isopropanol

2. Sonication in toluene for 20 minutes

3. Rinsing in isopropanol

4. Sonication in isopropanol for 20 minutes

5. Drying in a dust-free environment for 2 hours

Clean samples and counter surfaces were mounted in the rig and sufficient amount of dye containing lubricant added to the test chamber such that the contact was fully flooded (approx. 100 $\mathrm{ml}$ ). The test load was applied and images were captured at a series of entrainment speeds, beginning at a zero speed (static Hertzian contact) and increasing with exponential steps to 1200 $\mathrm{mm} / \mathrm{s}$. At the end of the test the contact was left at rest to squeeze out any remaining lubricant film and a further static image was then captured. A separate background image of a uniform lubricant film formed on a microscope slide was captured and used to calibrate for any spatial variations in the intensity of the illuminating laser. Each of the test images relating to a specific entrainment speed was normalised using this background image to remove any spatial error in illumination.

Calibration maps were obtained for all contact configurations by fitting the normalised intensity for the pre-test static contact to the theoretical film height obtained from the Hertz equation for the applied load, geometry and material properties of the elastomer and glass counter face. Calibration was performed for each tested sample. In order to ensure that there was no drift in the calibration over the test duration, the predicted film thickness for the final post-test static contact was checked and confirmed to be zero at the centre of the contact The film thickness map for each image was calculated by taking the pixel intensity values and multiplying by the calibrated film thickness to intensity relationship. A schematic of the calibration process is shown in Figure 3.

\subsection{Test Fluids}

The test fluid for all tests was $75 \%$ wt. glycerol, $25 \%$ wt. water solution containing $0.01 \%$ wt. Rhodamine $6 \mathrm{G}$ dye. The viscosity of the glycerol - water solution is $28.4 \mathrm{mPas}$ at $25^{\circ} \mathrm{C}$. It should be 
noted that the addition of a low percentage by weight of dye has no influence on the effective viscosity of the test fluid. All tests were conducted at the temperature of $25^{\circ} \mathrm{C}$.

\section{$5 \quad$ Results and Discussion}

For all results presented in this paper Figure 4 shows a schematic of the nominal contact configuration and the coordinate system used to plot the results. The coordinate system is fixed with respect to the centre of the field of view of the optical system and no realignment of the images was performed. Measurement uncertainty was calculated for the film thickness values using a formal statistical process which utilised the root sum squared method to compound error sources. This was estimated to be $\pm 121 \mathrm{~nm}$ or $1.5 \%$ of the measurand whichever was greater. This represents the uncertainty in the absolute values and should not be confused with the resolution of the method which was found to be in the region of $8 \mathrm{~nm}$.

\subsection{Configuration 1 - PDMS hemisphere on a flat disc (Point contact, $a / b=1$ )}

Maps of film thickness were obtained using the method described in section 3 for a PDMS hemisphere loaded against a flat glass disc in pure sliding and lubricated with a $75 \%$ wt. glycerol $25 \%$ wt. water solution containing $0.01 \%$ wt. Rhodamine $6 \mathrm{G}$ dye. Figures 5 and 6 show the contact film thickness maps recorded at four selected entrainment speeds and at applied load of $21 \mathrm{mN}$ and 11 $\mathrm{mN}$ respectively. It is evident that the present technique makes it possible to obtain good quality maps of film thickness across the entire contact area for a wide range of sliding speeds. The general circular form of the contact shape is clearly evident at low speed, $7.31 \mathrm{~mm} / \mathrm{s}$. As entrainment speed is increased to $113 \mathrm{~mm} / \mathrm{s}$ a distinct "crescent" shaped area of minimum film can be observed to form towards the rear of the contact. This constricted zone persists in similar form as entrainment speed increases from $113 \mathrm{~mm} / \mathrm{s}$ to $1190 \mathrm{~mm} / \mathrm{s}$. It does not seem to grow towards the inlet and there is no evidence of side-lobes that are usually present in standard EHL contacts.

The observed absence of side-lobes confirms previous theoretical predictions for I-EHL contacts [40]. It is also in line with previous measurements of Myant et al. [24] obtained at lower speeds using an optical interferometry technique. The current investigation confirms this observation over a wider range of speeds and film thicknesses.

Selected two-dimensional profiles of the film, along the $x$ axis at $y=0$, for a range of entrainment speeds and at applied contact loads of $11 \mathrm{mN}$ and $21 \mathrm{mN}$ are shown in Figures 7(a) and 7(b) respectively. The existence of the outlet constriction identified in Figure 5 can be clearly seen in the two dimensional film profiles, particularly at higher speeds. It is evident that the film thickness increases with increasing speed as may be expected, but the film shape also changes - in particular, the slope of the film in the entrainment direction increases significantly with increasing speed. This means that the central plateau region, associated with EHL, is completely absent and the resulting film shape is similar to a hydrodynamic wedge. This converging wedge has been observed by other authors [24, 41] while in Gasni et al. [36] experiments using ultrasonic reflections, the wedge was only prominent at relatively low speeds and the central film became flat at higher entrainment speeds.

The observed constriction at the contact exit is less pronounced here than that observed by Myant et al. [24] and Martin et al. [41]. This difference may be due to the fact that both previous studies considered a much lower range of speeds than the current study $(U=0.06 \mathrm{~mm} / \mathrm{s}$ to $1.4 \mathrm{~mm} / \mathrm{s}$ 
in the case of Myant et al. [24] and $U=0.01 \mathrm{~mm} / \mathrm{s}$ to $0.5 \mathrm{~mm} / \mathrm{s}$ in Martin's et al. [41] study) . In addition, Myant and co-authors also noted that the very sharp constriction that they observed may actually have been a consequence of the fact that they used a PDMS coated steel ball, rather than a solid PDMS ball, so that the thick PDMS layer could have been slipping on the steel substrate. The current experiments with a solid PDMS ball seem to confirm that, although an exit constriction does exist, it is not as sharp as indicated by the previous coated ball experiments.

In Figures $8(\mathrm{a})$ and $8(\mathrm{~b})$ the measured central film thickness is plotted against entrainment speed for a PDMS hemisphere against a glass flat at applied loads of $11 \mathrm{mN}$ and $21 \mathrm{mN}$ respectively. It should be noted that, given the existence of the central film plateau region, the central film thickness value is calculated from an average of $20 \times 20$ pixels located at the nominal centre of the static contact (i.e. at location of the largest transverse semi-width within the field of view). This procedure is followed for all central film thickness values reported in the current paper. A linear relationship between the lubricant film thickness and entrainment speed is seen when the measured data is plotted on a loglog plot. For comparison, the corresponding predictions for central film thickness from empirical models of Hamrock and Dowson [23], equation (1), and Nijenbanning et al. [25], equation (5), are shown on the same plots. It is evident that the measured film thicknesses are lower than those predicted by Hamrock and Dowson [23] but higher than Nijenbanning et al. [25] predictions, lying about half way between the two. The discrepancy between theoretical fits and current observations may, at least in part, be attributed to the fact that the theoretical predictions are based on empirical fits that cover a different range of speeds and loads than tested here. Hamrock and Dowson's solution was fitted to a narrow range of $\bar{U}$ and $\bar{W}$ values: $\bar{U}=5 \times 10^{-9}$ to $5 \times 10^{-8}$ and $\bar{W}=0.2$ to $2 \times 10^{-3}$. The current tests which cover the wider range $\bar{U}=2.6 \times 10^{-10}$ to $4.9 \times 10^{-7}$ and $\bar{W}=1.5 \times 10^{-5}$ to $2.6 \times 10^{-3}$ span the regions considered by Hamrock and Dowson. Interestingly, the film thickness values measured by Myant et al. were lower than Hamrock and Dowson predictions by about a factor of 2, which is a much larger discrepancy than that observed here. But their measurements were performed over speeds and loads that were even smaller, with $\bar{U}=9 \times 10^{-12}$ to $5 \times 10^{-9}$ and $\bar{W}=$ $3 \times 10^{-6}$ to $5 \times 10^{-5}$. Therefore, it would seem that the accuracy of the theoretical predictions is strongly influenced by the range of contact conditions considered.

Comparison of Figures $8(a)$ and $8(b)$ suggests that the contact load has a minimal influence on the measured central film thickness.

The observed formation of the inclined central region within the film, as evident in Figure 7(a), is worth considering further. Figure 9(a) shows the same results as Figure 7(a) but with the addition of lines of best fit for the inclined central wedge. If this part of the film profile is considered as a hydrodynamic wedge, it could be characterised by the convergence parameter, $k_{h y}=\left(h_{i}-h_{o}\right) / h_{o}$ [42], where $h_{i}$ and $h_{o}$ are the values of the film thickness at the inlet and the outlet respectively. If the points at which the film shape deviates significantly from the line of best fit are taken to represent the inlet and outlet, the equivalent values of $K_{h y}$ can be calculated for the current film profiles. These are shown for the selected speeds in Figure $9(a)$ and plotted against the complete range of speeds tested in Figure $9(\mathrm{~b})$. It can be seen that the calculated values of $K_{h y}$ increase rapidly at low entrainment speeds and seem to tend to a value around 1.3-1.4. Cameron [42] reports that for an inclined hydrodynamic pad bearing with a length to breadth ratio of 1 (i.e. of equal size in both the $x$ and y directions, which may be considered similar in extent to a point contact) the optimum convergence at which the load support is maximised for any given size, film thickness, viscosity or 
entrainment speed, lies between $K_{h y}=1.3$ and 1.4. Therefore, by comparison to the values predicted here, this would suggest that in the case of a PDMS hemisphere loaded against a glass flat studied here the contact shape behaves like a self-optimising hydrodynamic bearing, demonstrating increasing hydrodynamic efficiency as the entrainment speed increases. The observed increase in wedge angle with entrainment speed was also noted by Martin et al. [41], for a PDMS hemisphere lubricated with fluorinated silicone oil. In order to confirm the significance of the trends observed in the present study and the range of their applicability, further tests using different materials and contact conditions are required.

\subsection{Configuration 2 - FKM O-ring against a flat disc (Elliptical contact, $a / b=3.9$ )}

Maps of film thickness were obtained for an FKM O-ring loaded against a flat glass disc under pure rolling conditions and lubricated with a $75 \% \mathrm{wt}$. glycerol $25 \% \mathrm{wt}$. water solution containing $0.01 \%$ wt. Rhodamine $6 \mathrm{G}$ dye. Figures 10 shows maps of film thickness obtained at different entrainment speeds, at an applied static load of $5.4 \mathrm{~N}$. At $144 \mathrm{~mm} / \mathrm{s}$ minima in the film height can be observed at the side edges of the elliptical contact. Striations in the film thickness associated with the roughness of the seal material can clearly be seen. As entrainment speed increases these areas of film minima become smaller and more localised towards the outer edges of the nominal contact area. An area of thicker film is formed towards the centre of the contact with increasing entrainment speed. In Figure 11, two dimensional profiles are shown of the film along the $x$ axis at $y=0$, for a range of entrainment speeds and at applied contact load of $5.4 \mathrm{~N}$. Overall film thickness increases with increasing entrainment speed, with a clear maximum in the film thickness formed towards the middle of the contact. This maximum in film thickness becomes more pronounced as entrainment speed is increased to $1080 \mathrm{~mm} / \mathrm{s}$. The overall film does not form the type of "hydrodynamic wedge" seen for the non-conformal point contact considered in configuration 1 above and there does not seem to be any discernible reduction in film thickness towards the rear of the contact. In Figure 12, the measured central film thickness is plotted against entrainment speed for an FKM O-ring against a glass flat at applied load of $5.4 \mathrm{~N}$. As explained above, the reported central film thickness values are the average of a $20 \times 20$ pixel area located at the centre of the nominal static contact ellipse and in this configuration the centre of the contact ellipse is taken as the point at which the nominal contact ellipse is widest in the direction transverse to sliding. The log-log graph indicates an approximate linear relationship between the central lubricant film thickness and the entrainment speed. For comparison, the corresponding predictions for central film thickness from empirical models by Hamrock and Dowson [23], equation (1), and Nijenbanning et al. [25], equation (5), are shown on the same plots. The measured film thicknesses are closer in value to the predictions of Hamrock and Dowson than those of Nijenbanning et al., being slightly higher than Hamrock and Dowson's predictions. This is in contrast to the observations made for the point contact above, where the measured film values lie in between the two theoretical predictions.

\subsection{Configuration 3 - FKM O-ring against a concave lens (highly conformal elliptical contact $a / b=10.6)$}

Maps of film thickness were obtained for an FKM O-ring loaded against a concave cylindrical glass lens in pure siding and lubricated with a $75 \%$ wt. glycerol $25 \%$ wt. water solution containing $0.01 \%$ wt. Rhodamine $6 \mathrm{G}$ dye. Figures 13 and 14 show measured film thickness maps for loads of $3.6 \mathrm{~N}$ and $4.8 \mathrm{~N}$ respectively at selected entrainment speeds. The elliptical form of the contact shape may be seen at all entrainment speeds. Unlike in configuration 2 above, no clear areas of reduced film are seen at the sides of the ellipse. Instead, throughout the contact patch distributed areas of local 
minima in lubricant film thickness are present. These decrease in size as entrainment speed increases. The presence of these discrete minima suggests that the roughness of the elastomer sample dominates the film behaviour under the applied conditions. The average film thickness is low within the nominal contact area and the lubricant film remains below $1 \mu \mathrm{m}$ at all entrainment speeds tested. Selected profiles, along the $x$ axis at $y=0$, for selected entrainment speeds at applied contact loads of $3.6 \mathrm{~N}$ and $4.8 \mathrm{~N}$ respectively are shown in Figures 15(a) and (b). Overall surface lift off with increasing entrainment speed is low, as demonstrated by the small change in the measured lubricant film thickness at the inlet. However, within the nominal contact the mean lubricant film thickness increases with rising entrainment speed and complete separation is achieved for both loads at speeds over $53.5 \mathrm{~mm} / \mathrm{s}$. It can also be observed that the film thickness increases more rapidly with speed in the areas containing localised minima in film thickness than the areas which started off with appreciable film at low speeds. This may indicate that the roughness asperities are being increasingly compressed with rising entrainment speed, with likely formation of micro-films on asperity contacts. The roughness of the O-rings in this setup and the applied contact conditions are much more representative of those found in operating bearing seals than the two configurations studied above and the behaviour observed here offers some indication of the lubrication mechanisms that may be encountered in bearing seals. However, as discussed above, the contact studied here does have a small degree of non-conformity, which would serve to help entrain the lubricant into contact, unlike the nominally conformal contact of a rotary seal on a shaft. Further studies are therefore needed to reveal entrainment mechanisms in operating bearing seal contacts in particular.

In Figures 16(a) and 16(b), central film thickness (calculated as an average of the central 20x20 pixels as explained above) is plotted against entrainment speed for an applied load of $3.6 \mathrm{~N}$ and $4.8 \mathrm{~N}$ respectively. It is evident that lubricant film thickness increases linearly with entrainment speed when plotted on a log-log axis. Again, the empirical models for central film thickness by Hamrock and Dowson [23] and Nijenbanning et al. [25], are shown in addition to the measured data points for comparison. At high entrainment speeds, the measured film thicknesses are close to those predicted by Nijenbanning and co-authors. At lower entrainment speeds, below $50 \mathrm{~mm} / \mathrm{s}$, significant scatter is evident in the plotted film values and the match with theory is apparently poor. The reason for the observed scatter lies in the way the central film value is calculated - it is an average of central $20 \times 20$ pixel values and because surface roughness completely dominates the film at these low speeds, the calculated average will inevitably be strongly affected by the characteristics of the local roughness features captured in the images. Within this region, the plotted central film values represent an average of the thickness of lubricant pockets entrapped within the local roughness and cannot therefore be expected to follow the numerical predictions for full film I-EHL lubrication.

The relationship between the experimental data and theoretical predictions observed here, combined with those noted above for a sliding point contact (configuration 1 above) and the elliptical rolling contact (configuration 2 above) suggests that the accuracy of existing theoretical film predictions is strongly influenced by contact shape and applied contact conditions.

\subsection{Characteristics of the Applied Laser Induced Fluorescence Technique}

The described technique utilises an optimised optical set-up with a relatively high signal-to-noise ratio and was shown to be able to produce film thickness maps of the complete contact area and measure a very wide span of thicknesses, from $50 \mathrm{~nm}$ to $100 \mu \mathrm{m}$. This is a very large range of 
measurement and is one of the main reasons why the present technique is more suitable for I-EHL studies than optical interferometry. One significant limitation of a typical LIF set-up is the minimum thickness that can be resolved and this is often determined by the signal-to-noise ratio (SNR). In the present set-up a low SNR has been achieved through minimising the number of optical surfaces, higher quality lenses and filters and implementation of full epi-illumination. Consequently, the achievable lower limit of measurements for the present set-up is in the region $50 \mathrm{~nm}$ to $100 \mathrm{~nm}$, as is evident in the measurements performed with rough elastomers in contact configuration 3 (see Figures 12-15). This is a significant improvement on previous studies that used LIF for measuring film thickness in compliant contacts: Poll et al. [32] measured films down to $350 \mathrm{~nm}$ while Myant et al. [29] measured minimum thicknesses of $300 \mathrm{~nm}$. In those studies the SNR of the measurement provided the limiting factor. In principle, the SNR of the current optical setup is low enough to measure films considerably below the practical limit of $50 \mathrm{~nm}$ to $100 \mathrm{~nm}$. However, the practical limit here is imposed largely by the specimen quality and the requirement to calibrate each specimen using a static contact. When a static contact is used an uncertainty in the exact value of measured intensity that corresponds to the absence of fluorescent molecules is unknown and must be inferred from the comparison between the intensity and film shape obtained from Hertzian theory. The resulting zero offset dominates the measurement error in the current method.

\section{Conclusions}

A laser induced fluorescence technique was used to measure lubricant film thickness in compliant contacts under isoviscous elastic regime (I-EHL). A range of entrainment speeds and a selection of loads were studied for three different contact configurations: a point contact of a PDMS hemisphere on a glass disc in pure sliding, an elliptical contact $(a / b=3.9)$ of an FKM O-ring on a glass flat in pure rolling and a near-conformal contact $(a / b=10.6)$ of an FKM O-ring against a concave glass lens. The first two contact set-ups were chosen in order to provide further insight into fundamentals of I-EHL lubrication while the near-conformal sliding contact configuration is an attempt to more closely approximate the actual contact of a rotary seal and a shaft.

The presented LIF technique is able to produce clear film thickness maps of the whole contact. The optimised optical set-up results in a relatively high signal-to-noise ratio thus allowing the measurement of film thicknesses as low as $50 \mathrm{~nm}$. This compares favourably to previous studies of film thickness in compliant contacts using LIF techniques [29, 32].

In the case of PDMS point contact, the film shape was observed to exhibit a crescent shaped area of reduced film located towards the contact exit. Unlike in EHL contacts, no 'side lobes' of reduced film were observed. The two-dimensional profiles of the film along the $y=0$ line confirm the presence of the exit constriction and reveal the complete absence of the flat central region, the film instead assuming a shape of a converging wedge. If this central wedge is considered to be similar to a converging hydrodynamic pad, the convergence ratio $K_{h y}$, as used in hydrodynamic bearing theory can be calculated. $K_{h y}$ is found to tend to a value of 1.3 to 1.4 with increasing entrainment velocity. This is similar to the value for optimal load support in an inclined hydrodynamic pad bearing with breadth to length ratio of 1 [42]. A log-log plot shows a linear relationship between the film thickness and the entrainment speed. The test results were found to lie between the theoretical predictions of Hamrock and Dowson [23] and Nijenbanning et al. [25] 
The elliptical contact of an FKM O-ring against a glass flat under pure rolling conditions, exhibits a different film shape, where the central film is relatively flat while areas of minimum film are located very near the contact edges on either side of this flat region. Contact lift-off is clearly observed with increasing entrainment speed. Once again, the film thickness against entrainment speed shows a linear relationship on a log-log plot. In this case, however, the measured thicknesses are much closer to the theoretical predictions of Hamrock and Dowson [23] than those of Nijenbanning et al. [25]

The near conformal sliding contact of an FKM O-ring on a concave glass lens, which more closely represents the actual seal-shaft contact, does not show the areas of reduced film thickness on the sides of the contact. Film thickness maps reveal presence of discrete areas of low film thickness within the overall contact area. It is likely that these are caused by the relatively high roughness of the FKM O-ring and that their location corresponds to asperity peak contacts. With the increasing entrainment speed, some lift-off is observed at these reduced film areas while the overall contact lift off is minimal. This seems to suggest that roughness asperities are increasingly compressed by the hydrodynamic pressure build-up. Measured film thickness values show a linear trend with increasing entrainment speed on a log-log graph and fit the theoretical predictions of Nijenbanning et al. [25] better than those of Hamrock and Dowson [23].

The observed differences in the level of agreement between the test results and the theoretical predictions of either Hamrock and Dowson [23] or Nijenbanning et al. [25] suggest that the relative accuracy of these theoretical models is dependent on applied contact conditions and the contact shape in particular.

\section{$7 \quad$ Acknowledgements}

The authors gratefully acknowledge the support of SKF ERC for the work reported here and their permission to publish this paper.

\section{$8 \quad$ References}

[1] Ogata M, Fujii T, Shimotsuma Y. Study on fundamental characteristics of rotating lip-type oil seals. Proceedings of the 13th Leeds Lyon Symposium on Tribology, 1986, Series 11, Fluid Film Lubrication - Osborne Reynolds Centenary, 553-560, publ. Elsevier 1987.

[2] Gabelli A. Micro-elastohydrodynamic lubricant film formation in rotary lip seal contacts. Proceedings of the 15th Leeds-Lyon Symposium on Tribology, 1989, Series 14, Tribological Design of Machine Elements, 57-68, publ. Elsevier 1990.

[3] Roberts AD. Studies of lubricated rubber friction: Part 2: Optical techniques applied to practical problems. Tribology International. 1977;10:175-83.

[4] Fujii Y. Method for measuring transient friction coefficients for rubber wiper blades on glass surface. Tribology International. 2008;41:17-23.

[5] Pan XD. Significance of tuning bulk viscoelasticity via polymer molecular design on wet sliding friction of elastomer compounds. Tribology Letters. 2005;20:209-19. 
[6] Pan X. Wet sliding friction of elastomer compounds on a rough surface under varied lubrication conditions. Wear. 2007;262:707-17.

[7] Jin ZM, Dowson D. Elastohydrodynamic lubrication in biological systems. Proceedings of the Institution of Mechanical Engineers, Part J: Journal of Engineering Tribology. 2005;219:367-80.

[8] de Wijk RA, Prinz JF. The role of friction in perceived oral texture. Food Quality and Preference. 2005;16:121-9.

[9] Greenwood JA, Tabor D. The friction of hard sliders on lubricated rubber: The Importance of Deformation Losses. Proceedings of the Physical Society. 1958;71:989.

[10] Greenwood JA, Minshall H, Tabor D. Hysteresis losses in rolling and sliding friction. Proceedings of the Royal Society of London Series A, Mathematical and Physical Sciences. 1961;259:480-507.

[11] Grosch KA. The relation between the friction and visco-elastic properties of rubber. Proceedings of the Royal Society A: Mathematical, Physical and Engineering Sciences. 1963;274:21-39.

[12] Savkoor AR. On the friction of rubber. Wear. 1965;8:222-37.

[13] Roberts AD, Tabor D. Fluid film lubrication of rubber-an interferometric study. Wear. 1968;11:163-6.

[14] Roberts AD, Johnson KL. Lubrication of a rubber ball rolling with spin. Wear. 1974;27:225-35.

[15] Field GJ, Nau BS. A Theoretical study of the elastohydrodynamic lubrication of reciprocating rubber seals. ASLE Transactions. 1975;18:48-54.

[16] Roberts AD, Thomas AG. The adhesion and friction of smooth rubber surfaces. Wear. 1975;33:45-64.

[17] Moore DF. The elastohydrodynamic transition speed for spheres sliding on lubricated rubber. Wear. 1975;35:159-70.

[18] Roberts AD. Studies of lubricated rubber friction: Part 1: Coupling optical observations to friction measurements. Tribology International. 1977;10:115-22.

[19] Roberts AD. Surface charge contribution in rubber adhesion and friction. Journal of Physics D: Applied Physics. 1977;10:1801.

[20] Roberts $A D$, Parsons $A B$. Humidity influence on rolling friction of glass on rubber. Journal of Physics D: Applied Physics. 1979;12:1027.

[21] Jagger ET, Walker PS. Further studies of the lubrication of synthetic rubber rotary shaft seals. Proceedings of the Institution of Mechanical Engineers, 1966;181:191-204.

[22] Chittenden RJ, Dowson D, Dunn JF, Taylor CM. A theoretical analysis of the isothermal elastohydrodynamic lubrication of concentrated contacts. I. direction of lubricant entrainment coincident with the major axis of the Hertzian contact ellipse. Proceedings of the Royal Society of London A Mathematical and Physical Sciences. 1985;397:245-69. 
[23] Hamrock BJ, Dowson D. Elastohydrodynamic lubrication of elliptical contacts for materials of low elastic modulus I-fully fooded conjunction. Journal of Lubrication Technology. 1978;100:236.

[24] Myant C, Fowell M, Spikes HA, Stokes JR. An investigation of lubricant film thickness in sliding compliant contacts. Tribology Transactions. 2010;53:684-94.

[25] Nijenbanning G, Venner $\mathrm{CH}$, Moes H. Film thickness in elastohydrodynamically lubricated elliptic contacts. Wear. 1994;176:217-29.

[26] Crook A. The lubrication of rollers. Philosophical Transactions of the Royal Society of London Series A, Mathematical and Physical Sciences. 1958;250:387-409.

[27] Moes H. Optimum similarity analysis with applications to elastohydrodynamic lubrication. Wear. 1992;159:57-66.

[28] Poll G, Gabelli A. Formation of lubricant film in rotary sealing contacts: Part II---A new measuring principle for lubricant film thickness. Journal of Tribology. 1992;114:290-6.

[29] Myant C, Reddyhoff T, Spikes HA. Laser-induced fluorescence for film thickness mapping in pure sliding lubricated, compliant, contacts. Tribology International. 2010;43:1960-9.

[30] Smart AE, Ford RAJ. Measurement of thin liquid films by a fluorescence technique. Wear. 1974;29:41-7.

[31] Hoult DP, Takiguchi M. Calibration of the laser fluorescence technique compared with quantum theory. Tribology Transactions. 1991;34:440-4.

[32] Poll G, Gabelli A, Binnington PG, Qu J. Dynamic mapping of rotary lip seal lubricant films by fluorescent image processing. In: Nau BS, editor. Fluid Sealing: Springer Netherlands; 1992;55-77.

[33] Sugimura J, Hashimoto M, Yamamoto Y. Study of elastohydrodynamic contacts with fluorescence microscope. Tribology Series. 2000;38:609-17.

[34] Reddyhoff T, Choo JH, Spikes HA, Glovnea RP. Lubricant fow in an elastohydrodynamic contact Using fuorescence. Tribology Letters. 2010;38:207-15.

[35] Bongaerts JHH, Day JPR, Marriott C, Pudney PDA, Williamson AM. In situ confocal Raman spectroscopy of lubricants in a soft elastohydrodynamic tribological contact. Journal of Applied Physics. 2008;104:014913-10.

[36] Gasni D, Wan Ibrahim MK, Dwyer-Joyce RS. Measurements of lubricant film thickness in the isoviscous elastohydrodynamic regime. Tribology International. 2011;44:933-944.

[37] Miller JN, Group US. Standards in fluorescence spectrometry: Chapman and Hall; 1981.

[38] Selanger KA, Falnes J, Sikkeland T. Fluorescence lifetime studies of Rhodamine 6G in methanol. The Journal of Physical Chemistry. 1977;81:1960-3. 
[39] Bojarski, P. Temperature effect on the fluorescence spectra of two-component viscous solutions in the presence of forward and reverse nonradiative excitation energy transport. Journal of Luminescence. 1997;71(1):47-56

[40] de Vicente J, Stokes JR, Spikes HA. The frictional properties of Newtonian fluids in rolling-sliding soft-EHL contact. Tribology Letters. 2005;20:273-86.

[41] Martin A, Clain J, Buguin A, Brochard-Wyart F. Wetting transitions at soft, sliding interfaces. Physical Review E. 2002;65.

[42] Cameron A. Basic Lubrication Theory: Longman; 1971. 


\section{LIST OF TABLES}

Table 1: Details of parameters for each experimental configuration, all tests performed at $25^{\circ} \mathrm{C}$

\section{LIST OF FIGURES}

Figure 1 - Schematic of optical setup for laser induced fluorescence technique used for film thickness mapping

Figure 2: Schematics of film thickness measurement rig configurations

Figure 3: Schematic of calibration process for determining the fluorescence intensity to film thickness mapping

Figure 4: Nominal contact configuration within the field of view for all film thickness maps

Figure 5: Maps of lubricant film thickness at selected entrainment speeds for a PDMS hemisphere on a glass disk under pure sliding (configuration 1 ) at an applied load of $23.5 \mathrm{mN}$.

Figure 6: Maps of lubricant film thickness at selected entrainment speeds for a PDMS hemisphere on a flat glass disk under pure sliding (configuration 1 ) at an applied load of $11 \mathrm{mN}$.

Figure 7: Plot of lubricant film thickness along the $x$ axis at $y=0$ for a PDMS hemisphere on a flat glass disk under pure sliding (configuration 1), for selected entrainment speeds, at an applied load of (a) $23.5 \mathrm{mN}$ (b) $11 \mathrm{mN}$.

Figure 8: Plot of central film thickness (average of central 20x20 pixels) against entrainment speed for a PDMS hemisphere on a flat glass disk under pure sliding (configuration 1) at an applied load of (a) $23.5 \mathrm{mN}$ (b) $11 \mathrm{mN}$.

Figure 9: (a) Plot of lubricant film thickness along the $x$ axis at $y=0$ for a PDMS hemisphere on glass disk (configuration 1), for a selection of speeds, at an applied load of $23.5 \mathrm{mN}$. Lines of best fit (dashed lines, ---) shown for inclined central film regions with associated values of convergence parameter $K,\left(K=\left(h_{i}-h_{0}\right) / h_{o}\right)$; (b) Plot of convergence parameter against entrainment speed

Figure 10: Maps of lubricant film thickness at selected entrainment speeds for a FKM O-ring on a flat glass disc under pure rolling conditions (configuration 2) at an applied load of 5.4N

Figure 11: Plot of lubricant film thickness along the $x$ axis at $y=0$ for a FKM O-ring on a flat glass disc under pure rolling conditions (configuration 2) at selected entrainment speeds and an applied load of $5.4 \mathrm{~N}$.

Figure 12: Plot of central film thickness (average of central 20x20 pixels) against entrainment speed for a FKM O-ring on a flat glass disc under pure rolling conditions (configuration 2) at an applied load of $5.4 \mathrm{~N}$.

Figure 13: Maps of lubricant film thickness at selected entrainment speeds for an FKM O-ring on a cylindrical glass lens in pure sliding (configuration 3 ) at an applied load of $4.8 \mathrm{~N}$ 
Figure 14: Maps of lubricant film thickness at selected entrainment speeds for an FKM O-ring on a cylindrical glass lens in pure sliding (configuration 3 ) at an applied load of $3.6 \mathrm{~N}$

Figure 15: Plot of lubricant film thickness along the $x$ axis at $y=0$ for a FKM O-ring on a cylindrical glass lens in pure sliding (configuration 3 ) at selected entrainment speeds, at an applied load of (a) $4.8 \mathrm{~N}(\mathrm{~b}) 3.6 \mathrm{~N}$.

Figure 16: Plot of central film thickness (average of central 20x20 pixels) against entrainment speed for an FKM O-ring sliding against a cylindrical glass lens (configuration 3) at an applied load of (a) $4.8 \mathrm{~N}(\mathrm{~b}) 3.6 \mathrm{~N}$. 


\begin{tabular}{|c|c|c|c|c|c|c|c|c|c|}
\hline \multirow[b]{2}{*}{ Configuration } & \multicolumn{2}{|l|}{ Test Conditions } & \multicolumn{4}{|c|}{ Contact Parameters } & \multicolumn{3}{|c|}{ Elastomer Parameters } \\
\hline & $\begin{array}{l}\text { Entrainment } \\
\text { Speed }\left(\mathrm{mm} \mathrm{s}^{-1}\right)\end{array}$ & $\begin{array}{l}\text { Load } \\
(\mathrm{mN})\end{array}$ & $\begin{array}{l}R_{x}^{\prime} \\
(\mathrm{mm})\end{array}$ & $\begin{array}{l}a_{\text {hertz }} \\
/ \\
b_{\text {hertz }}\end{array}$ & $\begin{array}{l}P_{\text {hertz }} \\
(\mathrm{kPa})\end{array}$ & $\begin{array}{l}a_{\text {hertz }} \\
(\mu \mathrm{m})\end{array}$ & Type & $\begin{array}{l}\mathrm{E} \\
\text { (MPa) }\end{array}$ & $\begin{array}{l}R_{a} \\
(n m)\end{array}$ \\
\hline $\begin{array}{l}1(\mathrm{a}) \\
\text { (Hemisphere } \\
\text { on Flat) }\end{array}$ & $\begin{array}{l}2.4 \text { to } 1190 \\
\text { (Sliding) }\end{array}$ & 23.5 & 12.7 & 1 & 89 & 355 & PDMS & 3.8 & $<10$ \\
\hline $\begin{array}{l}\text { 1(b) } \\
\text { (Hemisphere } \\
\text { on Flat) }\end{array}$ & $\begin{array}{l}2.4 \text { to } 221 \\
\text { (Sliding) }\end{array}$ & 11 & 12.7 & 1 & 69 & 275 & PDMS & 3.8 & $<10$ \\
\hline $\begin{array}{l}2 \\
\text { (O-Ring on } \\
\text { Flat) }\end{array}$ & $\begin{array}{l}5.5 \text { to } 1080 \\
\text { (Rolling) }\end{array}$ & 5400 & 10.4 & 3.9 & 2100 & 2178 & FKM & 7.3 & $\begin{array}{l}800 \\
50\end{array}$ \\
\hline $\begin{array}{l}3(\mathrm{a}) \\
\text { (O-Ring on } \\
\text { Concave } \\
\text { Lens) }\end{array}$ & $\begin{array}{l}9.8 \text { to } 105 \\
\text { (Sliding) }\end{array}$ & 4800 & 50.2 & 10.6 & 1400 & 4144 & FKM & 7.3 & $\begin{array}{l}800 \\
50\end{array}$ \\
\hline $\begin{array}{l}3(\mathrm{~b}) \\
\text { (O-Ring on } \\
\text { Concave } \\
\text { Lens) }\end{array}$ & $\begin{array}{l}9.8 \text { to } 105 \\
\text { (Sliding) }\end{array}$ & 3630 & 50.2 & 10.6 & 1300 & 3765 & FKM & 7.3 & $\begin{array}{l}800 \\
50\end{array}$ \\
\hline
\end{tabular}

Table 1: Details of parameters for each experimental configuration, all tests performed at $25^{\circ} \mathrm{C}$ 


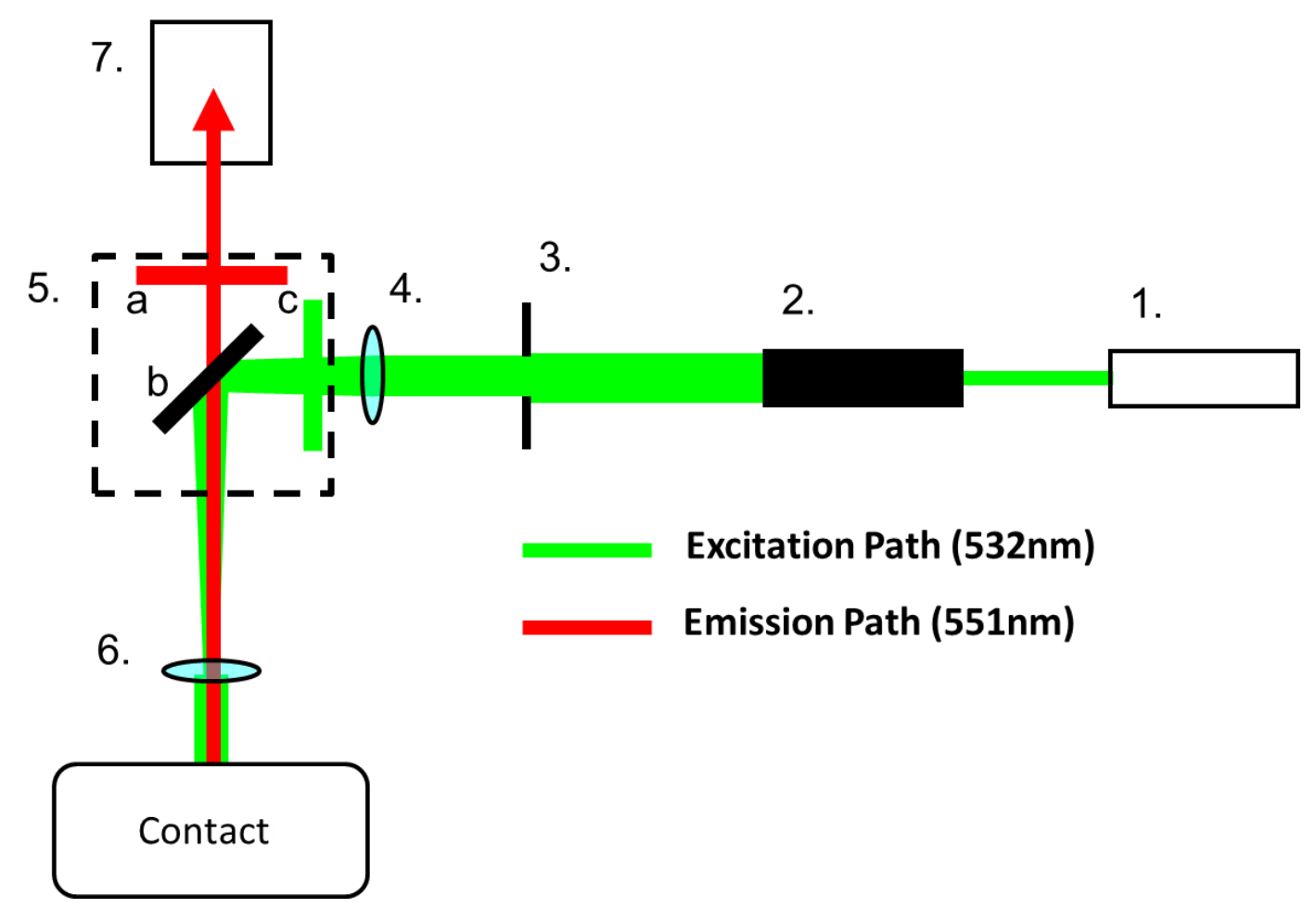

\begin{tabular}{|ll|ll|}
\hline 1. & CNi MLL-III Diode Laser (532nm, 400mW) & 2. & ThorLabs 10X Galilean Beam Expander \\
3. & Field Stop & 4. & Focusing Plano-Convex Lens \\
5. & Filter Cube & $5(a)$. & Emission Filter (Chroma, USA) \\
5(b). & Dichroic Mirror (Chroma, USA) & $5(c)$. & Excitation Filter (Chroma, USA) \\
6. & Zeiss Neofluar 5x Objective & 7. & QImaging Rolera MGi EMCCD Camera \\
\hline
\end{tabular}

Figure 1 - Schematic of optical setup for laser induced fluorescence technique used for film thickness mapping 

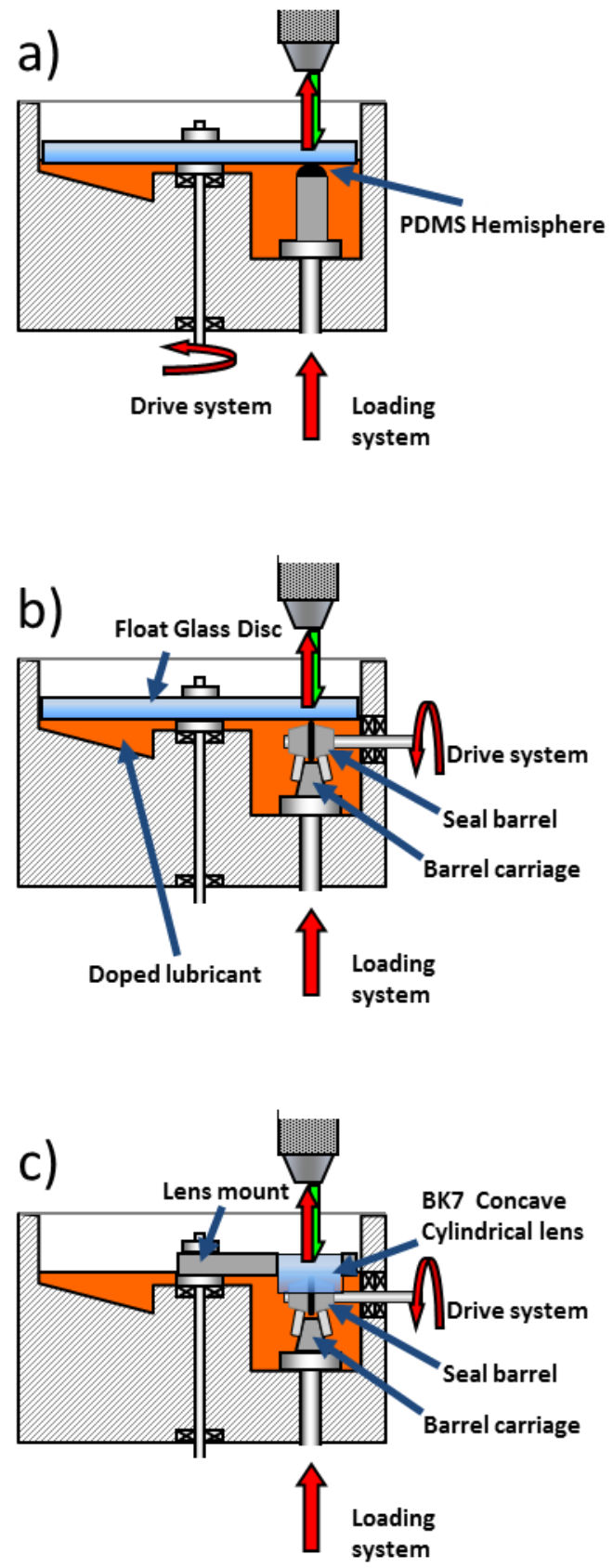

Figure 2: Schematics of film thickness measurement rig configurations 


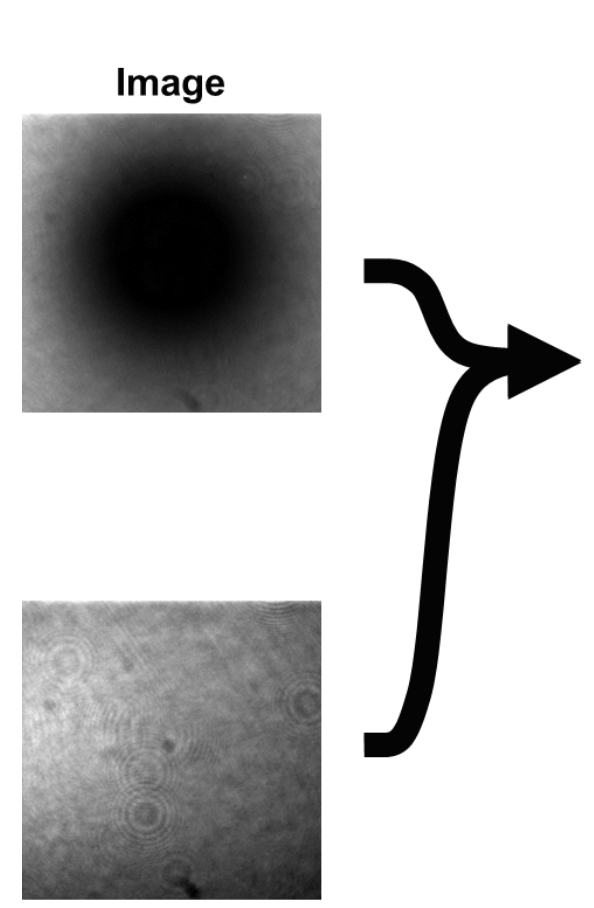

Background
Normalised
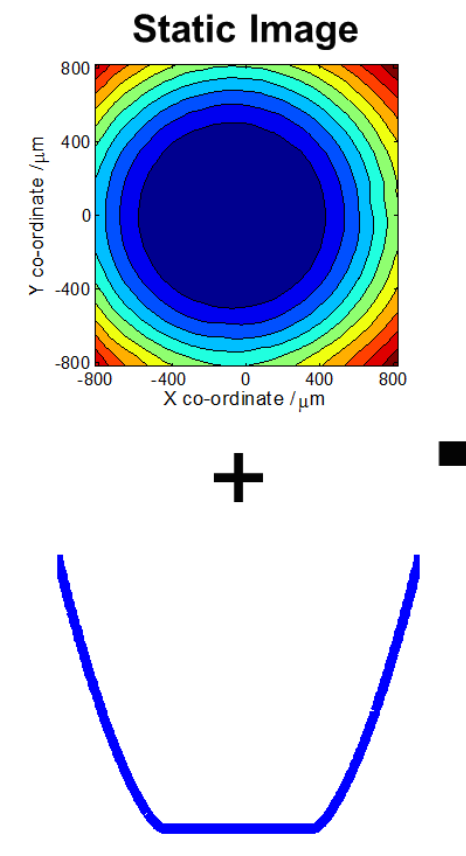

Hertz Theory

Figure 3: Schematic of calibration process for determining the fluorescence intensity to film thickness mapping 


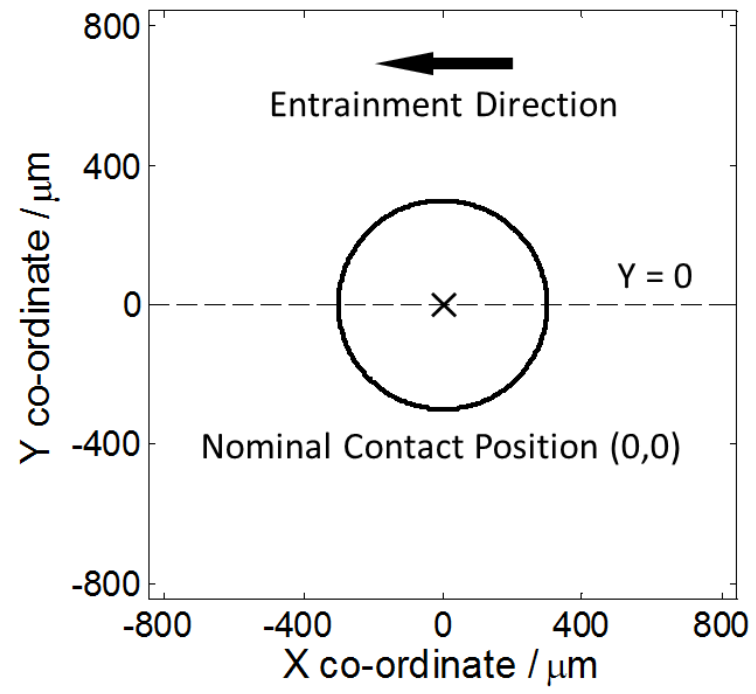

Figure 4: Nominal contact configuration within the field of view for all film thickness maps 

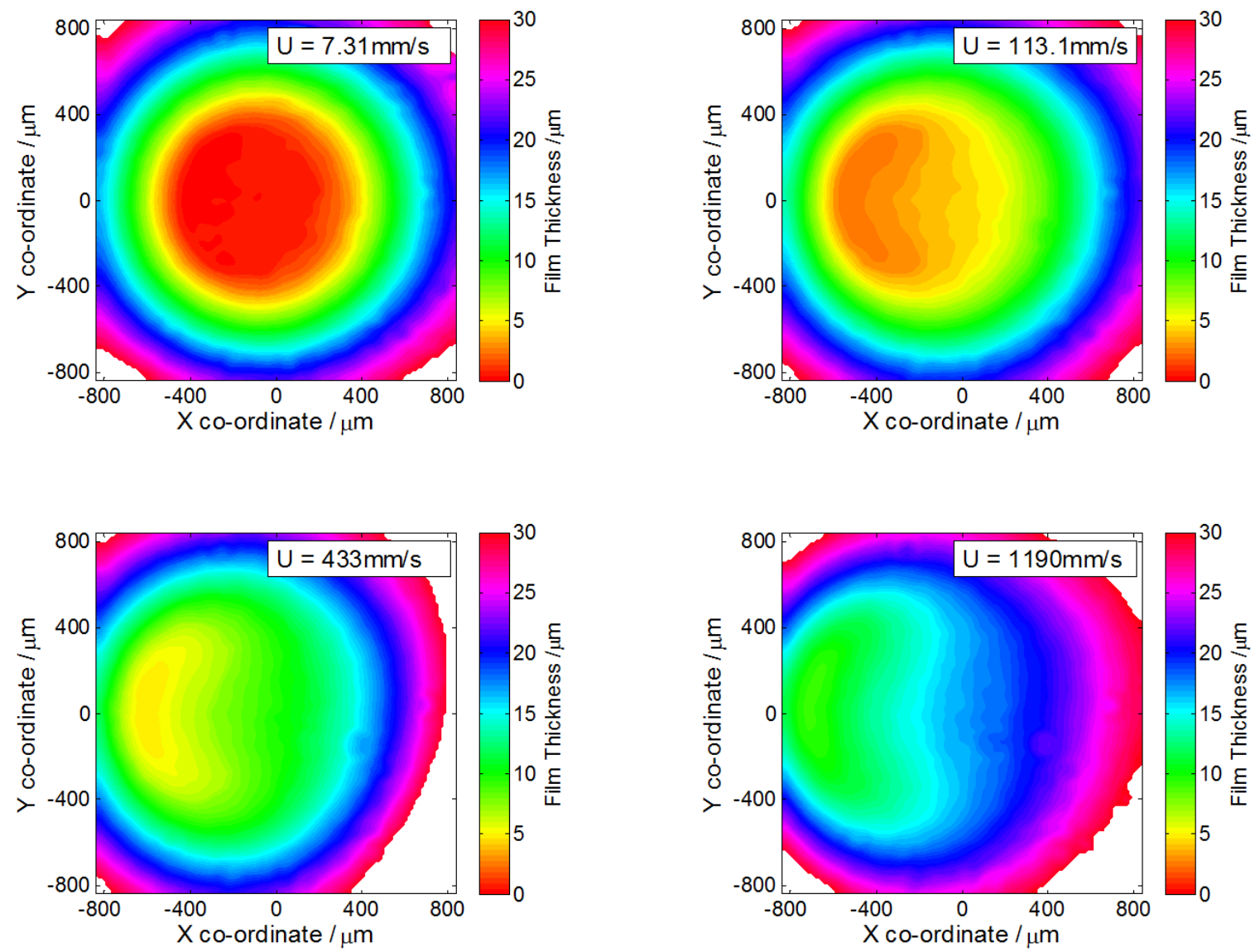

Figure 5: Maps of lubricant film thickness at selected entrainment speeds for a PDMS hemisphere on a glass disk under pure sliding (configuration 1 ) at an applied load of $23.5 \mathrm{mN}$. 

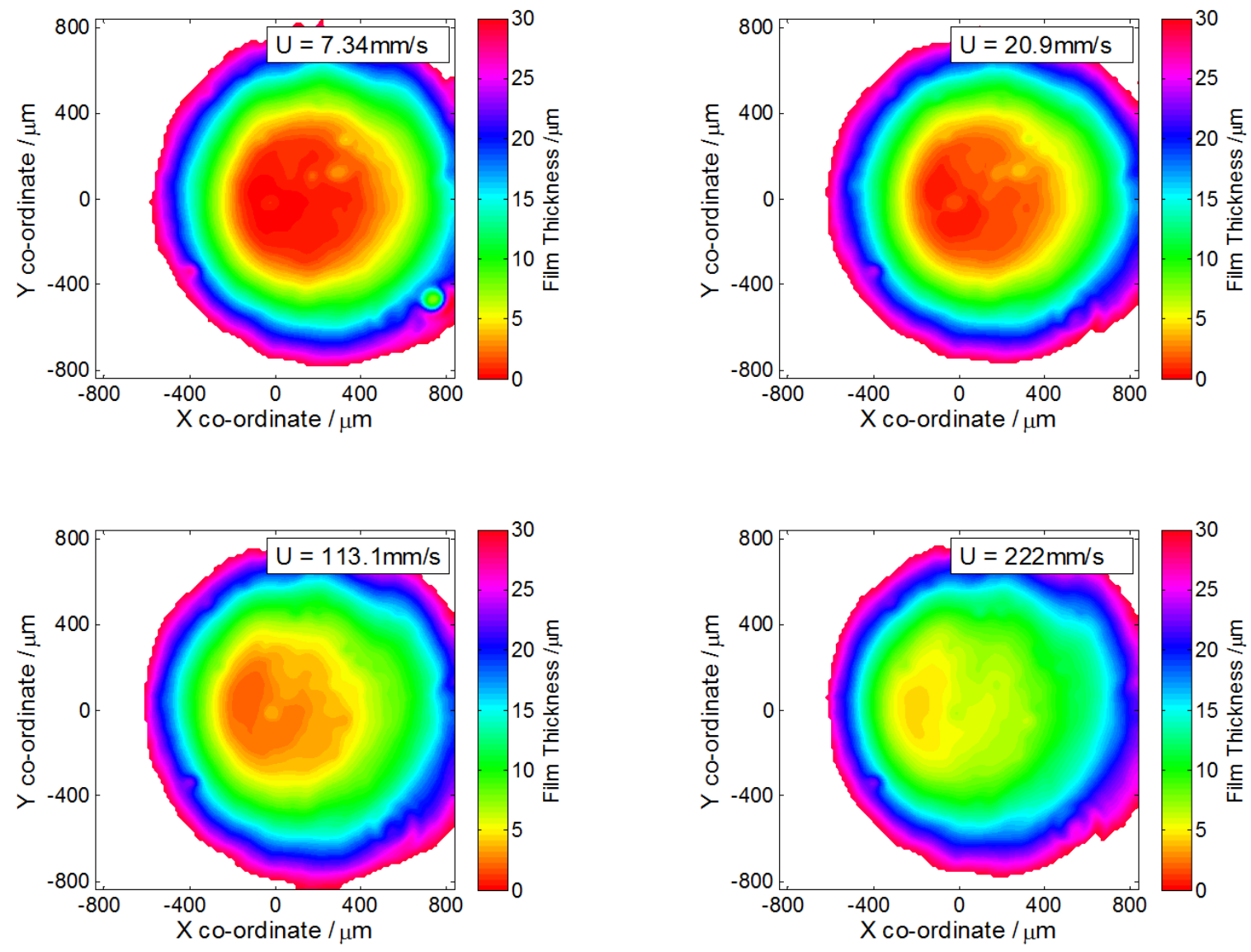

Figure 6: Maps of lubricant film thickness at selected entrainment speeds for a PDMS hemisphere on a flat glass disk under pure sliding (configuration 1 ) at an applied load of $11 \mathrm{mN}$. 

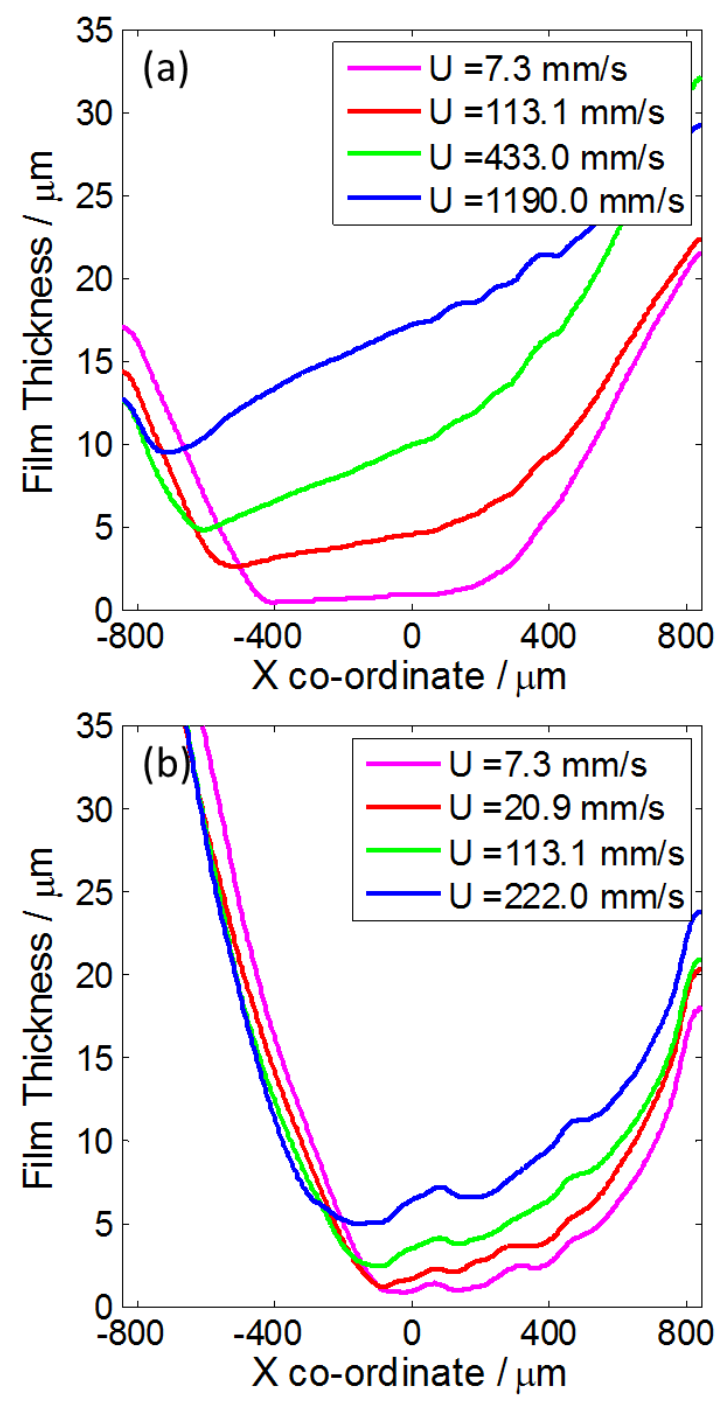

Figure 7: Plot of lubricant film thickness along the $x$ axis at $y=0$ for a PDMS hemisphere on a flat glass disk under pure sliding (configuration 1), for selected entrainment speeds, at an applied load of (a) $23.5 \mathrm{mN}$ (b) $11 \mathrm{mN}$. 

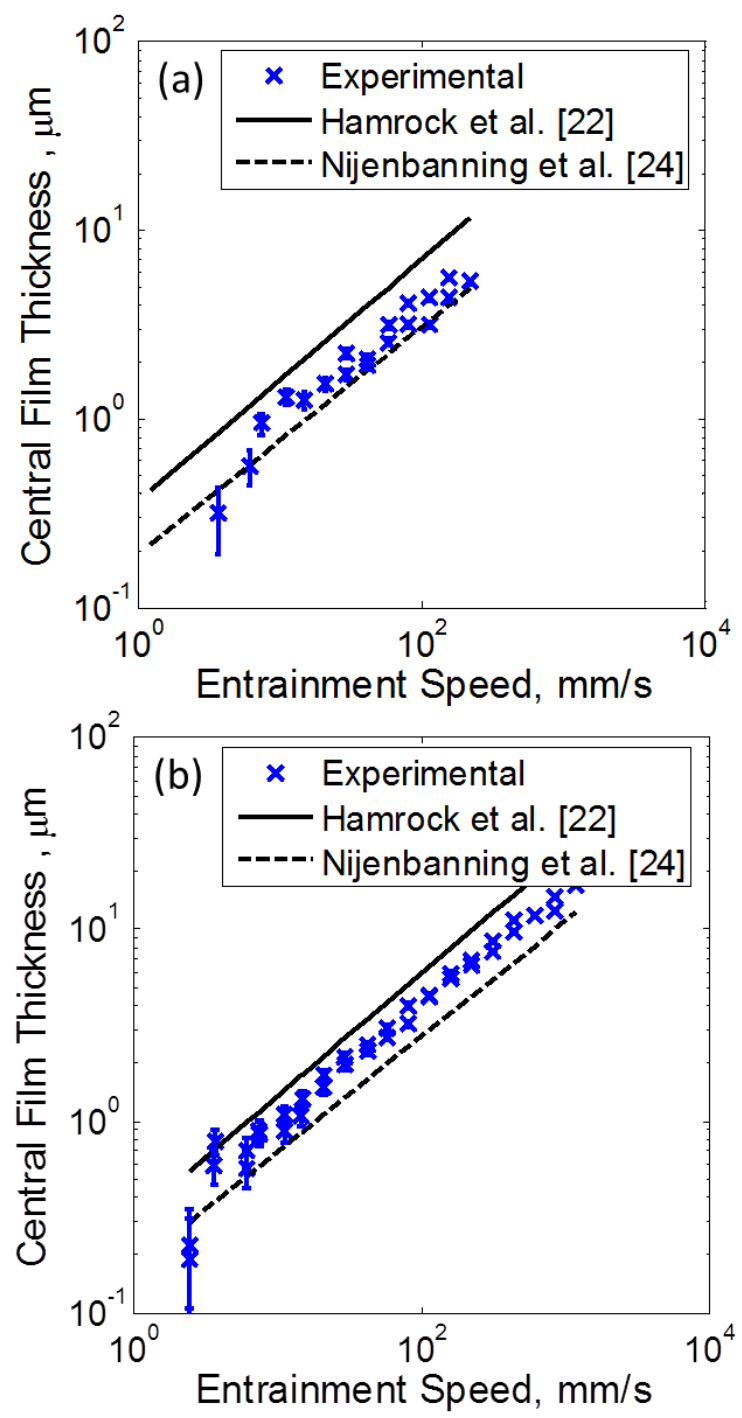

Figure 8: Plot of central film thickness (average of central 20x20 pixels) against entrainment speed for a PDMS hemisphere on a flat glass disk under pure sliding (configuration 1) at an applied load of (a) $23.5 \mathrm{mN}$ (b) $11 \mathrm{mN}$. 

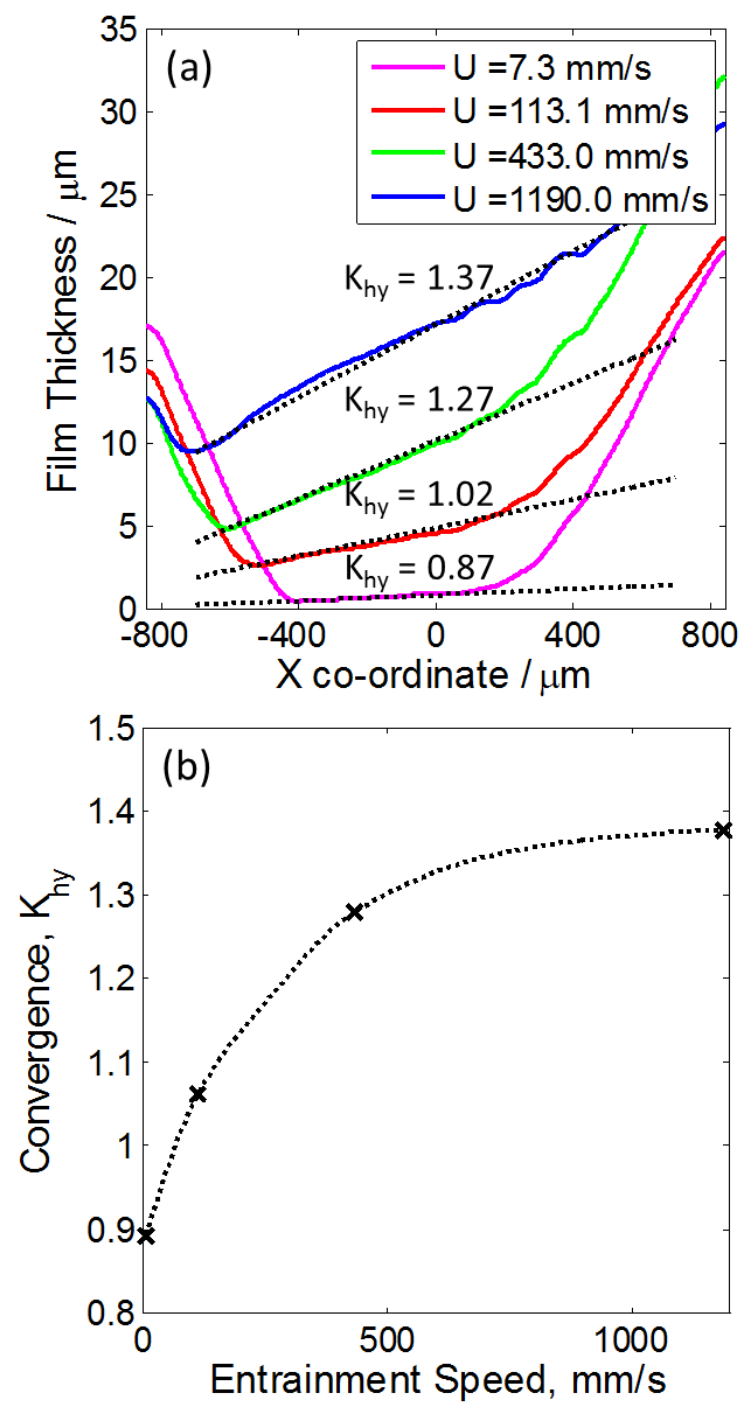

Figure 9: (a) Plot of lubricant film thickness along the $x$ axis at $y=0$ for a PDMS hemisphere on glass disk (configuration 1), for a selection of speeds, at an applied load of $23.5 \mathrm{mN}$. Lines of best fit (dashed lines, ---) shown for inclined central film regions with associated values of convergence parameter $K_{h y}\left(K_{h y}=\left(h_{i}-h_{0}\right) / h_{o}\right)$; (b) Plot of convergence parameter against entrainment speed 

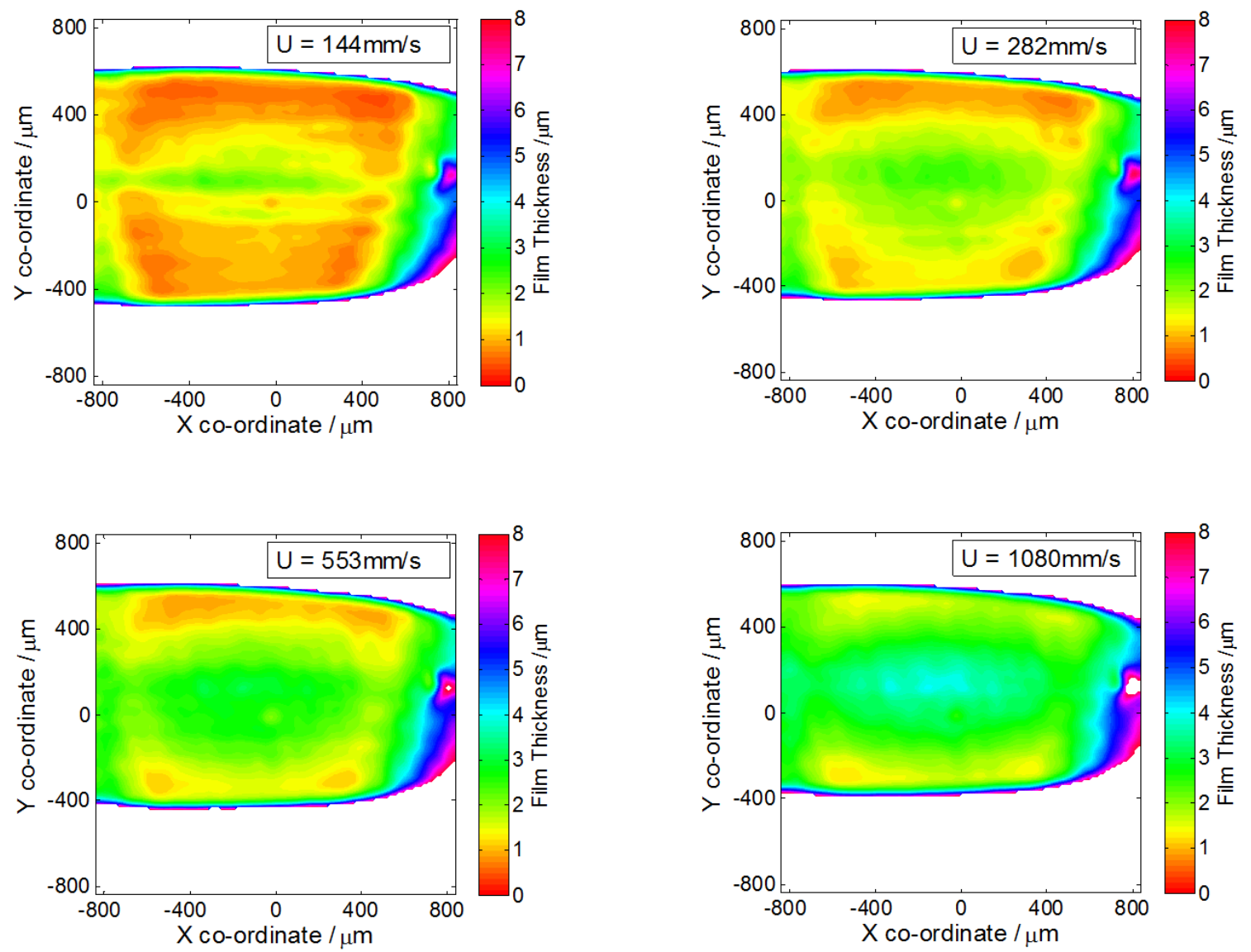

Figure 10: Maps of lubricant film thickness at selected entrainment speeds for a FKM O-ring on a flat glass disc under pure rolling conditions (configuration 2) at an applied load of $5.4 \mathrm{~N}$ 


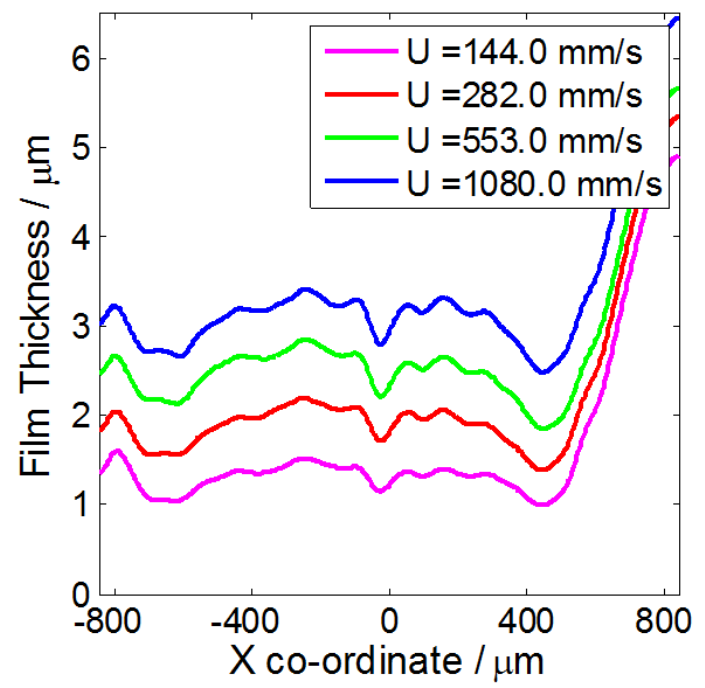

Figure 11: Plot of lubricant film thickness along the $x$ axis at $y=0$ for a FKM O-ring on a flat glass disc under pure rolling conditions (configuration 2 ) at selected entrainment speeds and an applied load of $5.4 \mathrm{~N}$. 


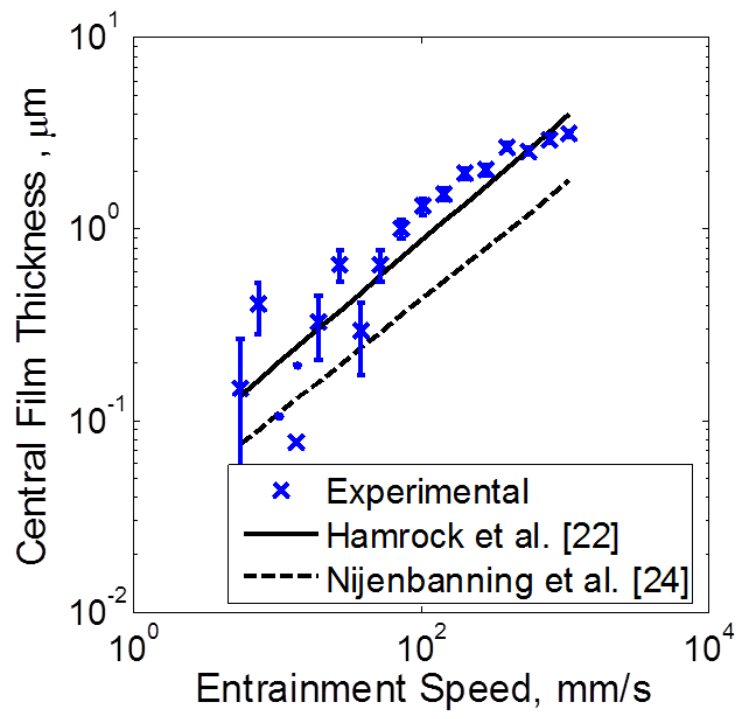

Figure 12: Plot of central film thickness (average of central 20x20 pixels) against entrainment speed for a FKM O-ring on a flat glass disc under pure rolling conditions (configuration 2) at an applied load of $5.4 \mathrm{~N}$. 

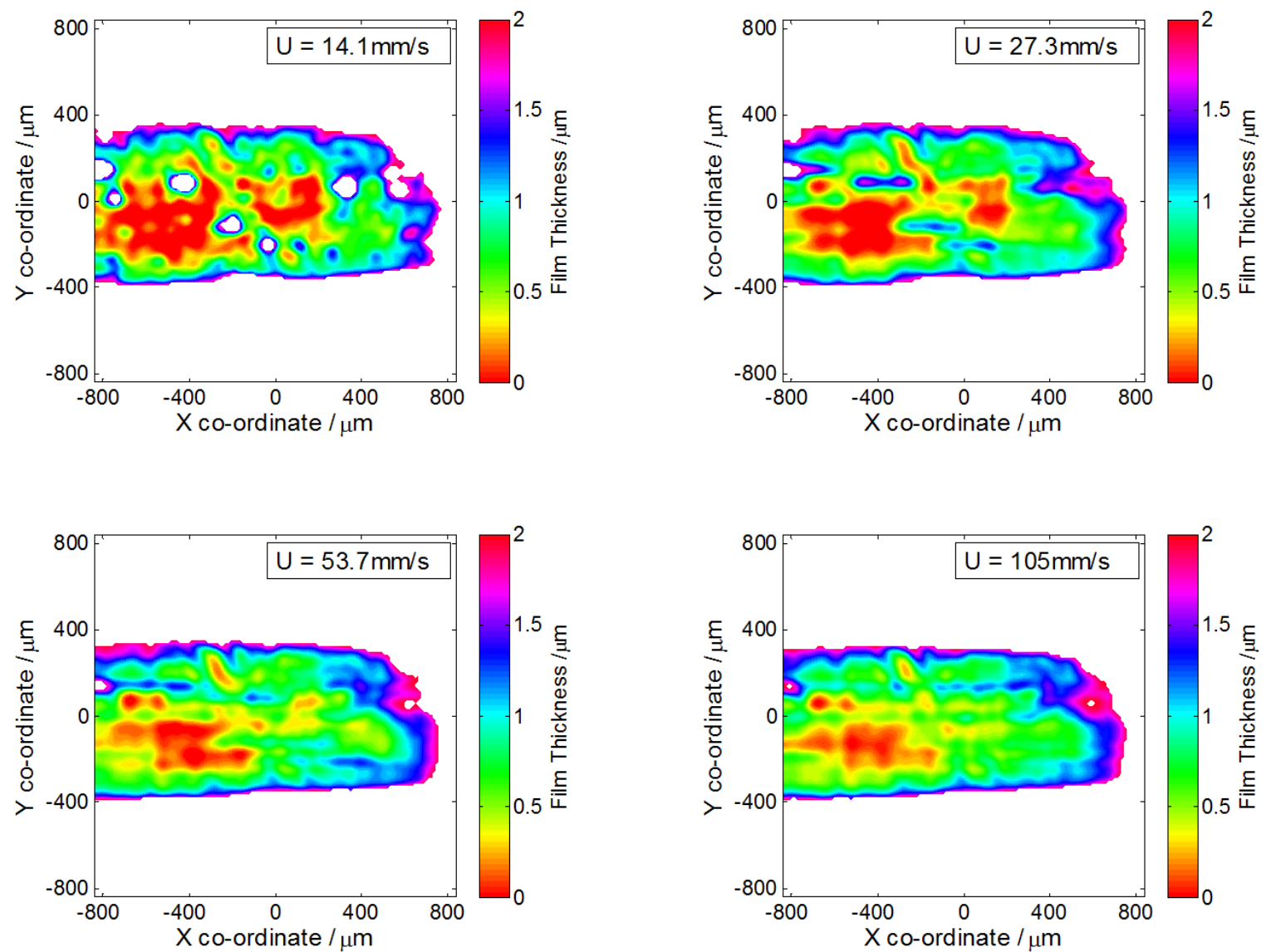

Figure 13: Maps of lubricant film thickness at selected entrainment speeds for an FKM O-ring on a cylindrical glass lens in pure sliding (configuration 3 ) at an applied load of $4.8 \mathrm{~N}$ 

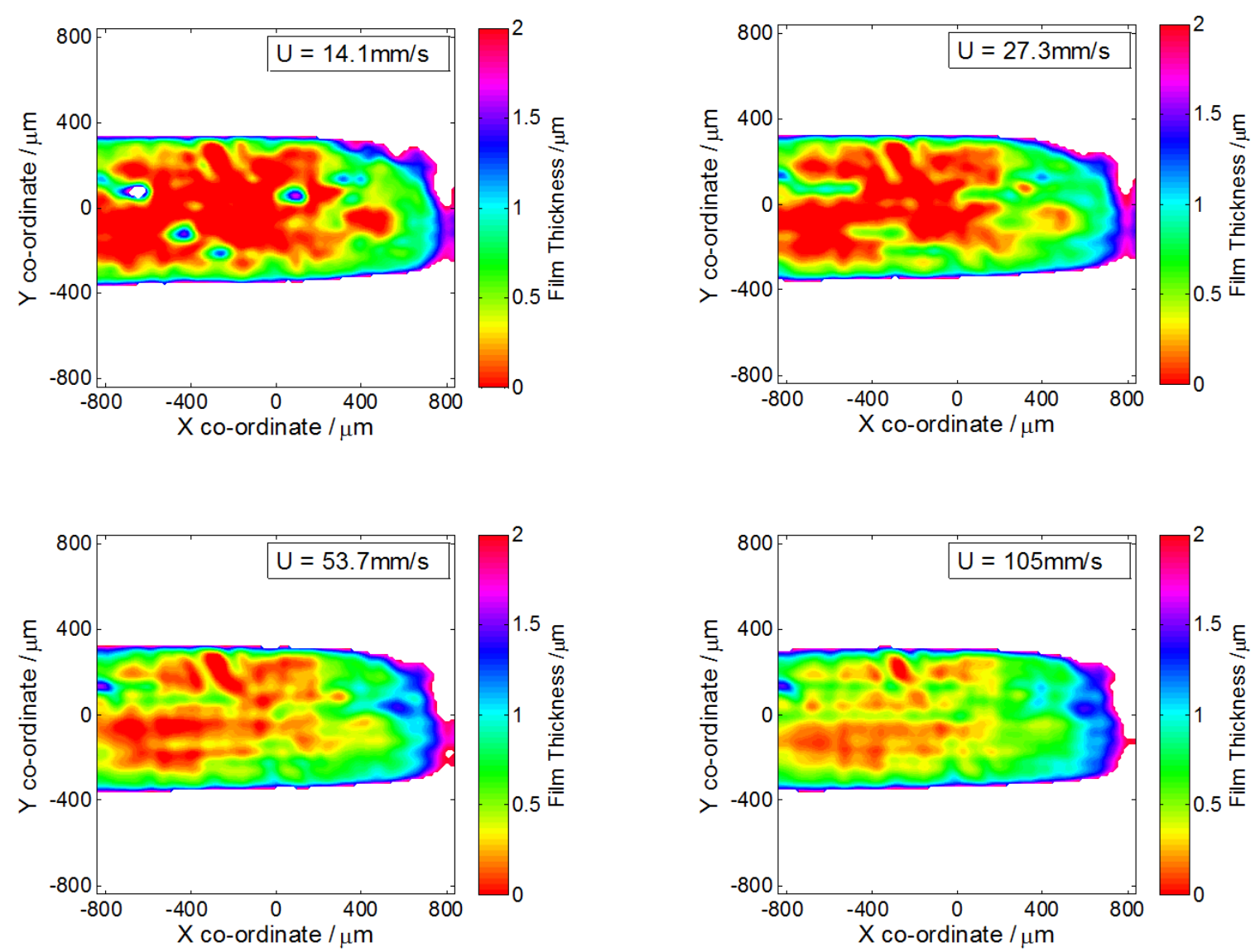

Figure 14: Maps of lubricant film thickness at selected entrainment speeds for an FKM O-ring on a cylindrical glass lens in pure sliding (configuration 3 ) at an applied load of $3.6 \mathrm{~N}$ 

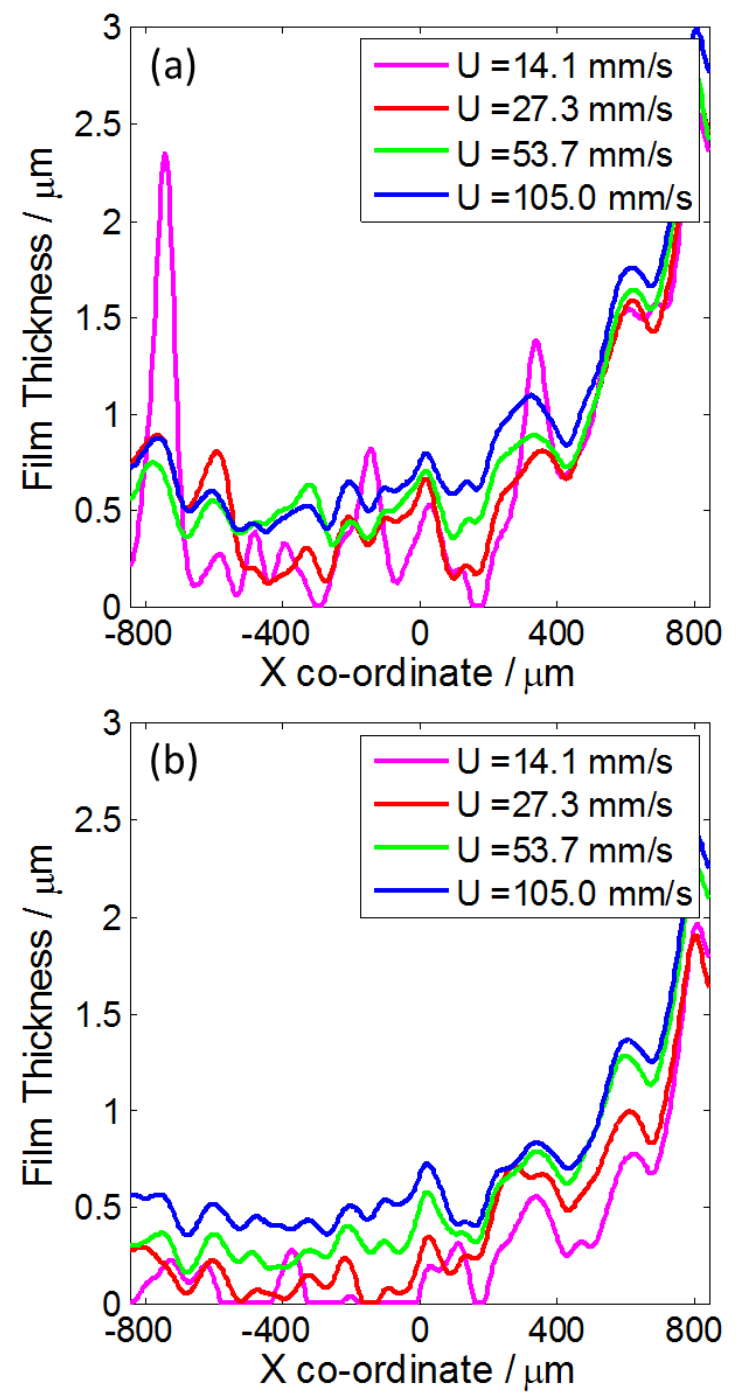

Figure 15: Plot of lubricant film thickness along the $x$ axis at $y=0$ for a FKM O-ring on a cylindrical glass lens in pure sliding (configuration 3 ) at selected entrainment speeds, at an applied load of (a) $4.8 \mathrm{~N}(\mathrm{~b}) 3.6 \mathrm{~N}$. 

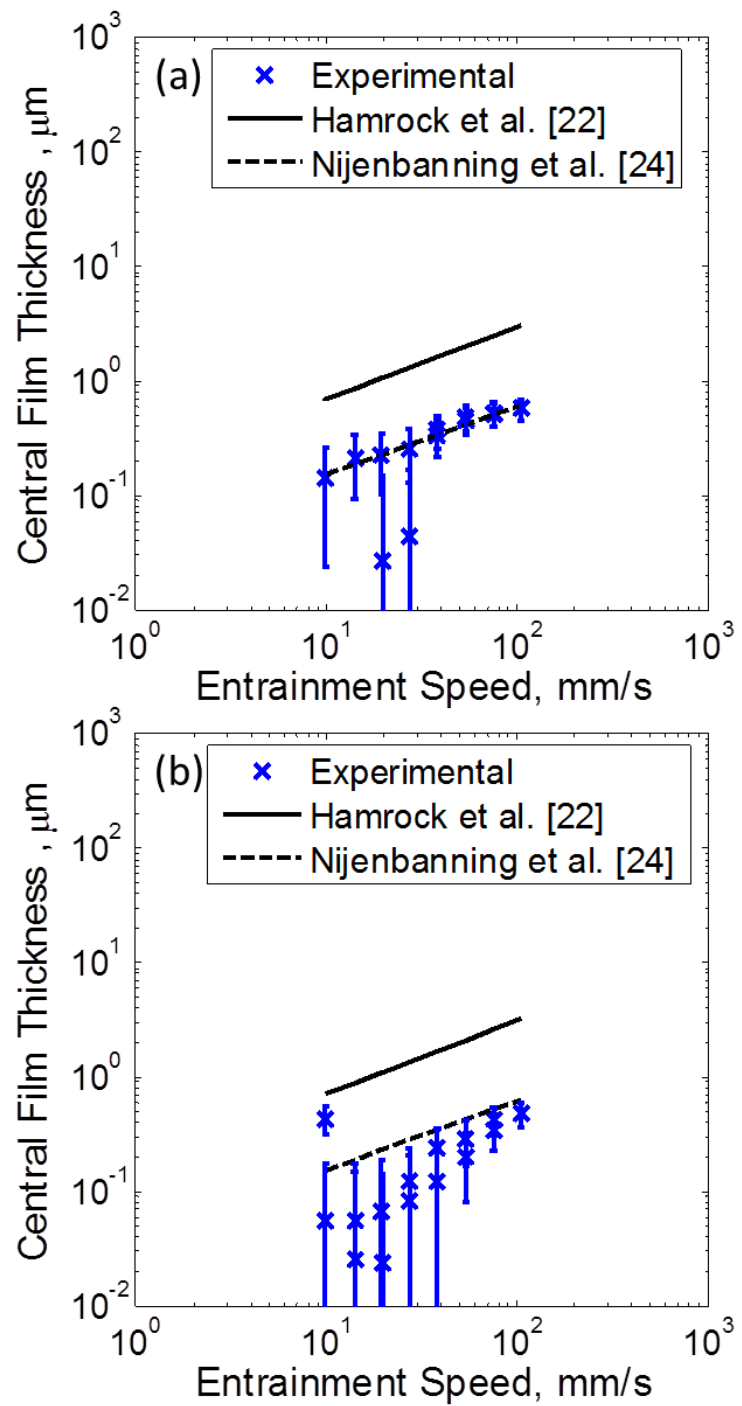

Figure 16: Plot of central film thickness (average of central 20x20 pixels) against entrainment speed for an FKM O-ring sliding against a cylindrical glass lens (configuration 3) at an applied load of (a) $4.8 \mathrm{~N}(\mathrm{~b}) 3.6 \mathrm{~N}$. 